\title{
Genomics of parallel ecological speciation in Lake Victoria cichlids
}

Joana Isabel MEIER (JIM) $)^{1,2,3}$, David Alexander MARQUES (DAM) $)^{1,2,3}$, Catherine Elise WAGNER $(C E W)^{1,3,5}$, Laurent EXCOFFIER (LE) $)^{2,4}$ \& Ole SEEHAUSEN $(\mathrm{OS})^{1,3, *}$

${ }^{1}$ Aquatic Ecology and Evolution, Institute of Ecology and Evolution, University of Bern, Baltzerstrasse 6, CH-3012 Bern, Switzerland; ${ }^{2} \mathrm{CMPG}$, Institute of Ecology and Evolution, University of Bern, Baltzerstrasse 6, CH-3012 Bern, Switzerland; ${ }^{3}$ Department of Fish Ecology and Evolution, EAWAG Swiss Federal Institute of Aquatic Science and Technology, Center for Ecology, Evolution and Biogeochemistry, Seestrasse 79, CH-6047 Kastanienbaum, Switzerland; ${ }^{4}$ Swiss Institute of Bioinformatics, CH-1015 Lausanne, Switzerland; ${ }^{5}$ Current address: Biodiversity Institute \& Botany Department, University of Wyoming, Laramie, WY USA

*Corresponding author (ole.seehausen@eawag.ch)

\begin{abstract}
The genetic basis of parallel evolution of similar species is of great interest in evolutionary biology. In the adaptive radiation of Lake Victoria cichlid fishes, sister species with either blue or red-back male nuptial coloration have evolved repeatedly, often associated with shallower and deeper water, respectively. One such case are blue and red-backed Pundamilia species, for which we recently showed that a young species pair may have evolved through "hybrid parallel speciation". Coalescent simulations suggested that the older species $P$. pundamilia (blue) and P. nyererei (red-back) admixed in the Mwanza Gulf and that new "nyererei-like" and "pundamilia-like" species evolved from the admixed population. Here, we use genome scans to study the genomic architecture of differentiation, and assess the influence of hybridization on the evolution of the younger species pair. For each of the two species pairs, we find over 300 genomic regions, widespread across the genome, which are highly differentiated. A subset of the most strongly differentiated regions of the older pair are also differentiated in the younger pair. These shared differentiated regions often show parallel allele frequency differences, consistent with the hypothesis that admixture-derived alleles were targeted by divergent selection in the hybrid population. However, two thirds of the genomic regions that are highly differentiated between the younger species are not highly differentiated between the older species, suggesting independent evolutionary responses to selection pressures. Our analyses reveal how divergent selection on admixture-derived genetic variation can facilitate new speciation events.
\end{abstract}

(C) The Author(s) 2018. Published by Oxford University Press on behalf of the Society for Molecular Biology and Evolution.

All rights reserved. For permissions, please e-mail: journals.permissions@oup.com 


\section{Introduction}

Understanding the genetic underpinnings of reproductive isolation and divergent adaptation is a major focus in speciation research. Some verbal models of speciation-with-gene-flow predict that genomic divergence in the face of gene flow is initially confined to very few small genomic regions under strong divergent selection (Wu 2001; Feder, et al. 2012a; Via 2012). One idea is that diverging regions may eventually grow due to local reduction of gene flow, allowing nearby sites under weaker divergent selection to diverge too (Via and West 2008; Feder and Nosil 2010; Feder, et al. 2012a; Via 2012). As the regions of divergence increase in number and size, genome-wide reduction of gene flow eventually results (Barton 1983; Wu 2001; Feder, et al. 2012a; Feder, et al. 2012b; Flaxman, et al. 2012; Flaxman, et al. 2014). Taxa at early stages of speciation that still hybridize are particularly suitable study systems for speciation-with-gene-flow as they have not yet accumulated differences that arose after reproductive isolation is complete (Coyne and Orr 2004). Ideally, one would study replicate pairs of taxa that differ in nothing else but strength of reproductive isolation, in order to assess the factors influencing variation in the cessation of gene flow between emerging species (Martin, et al. 2013; Riesch, et al. 2017). Pairs of species that have evolved through parallel speciation offer such an opportunity, and have provided compelling evidence for a role of selection in speciation (Schluter and Nagel 1995; Rundle, et al. 2000; Nosil, et al. 2009; Johannesson, et al. 2010).

In the young adaptive radiation of Lake Victoria cichlid fishes, replicates of similar species pairs are characterized by parallel divergence along the same major phenotypic and ecological axes (Seehausen 1996). Altogether the radiation comprises about 500 species (Seehausen 2002) that likely evolved in less than 15,000 years (Johnson, et al. 2000; Stager and Johnson 2008). The evolution of hundreds of phenotypically and ecologically distinct species in rapid succession requires large amounts of genetic variation in traits relevant to ecological adaptation and reproductive isolation. In an earlier study (Meier, et al. 2017a), we showed that an ancient admixture event between two divergent cichlid lineages, providing such genetic variation, preceded the adaptive radiations of cichlids in Lake Victoria and other lakes in the region. During the process of adaptive radiation, species may fix different adaptive variants and thus lose genetic variation at sites conferring adaptation to different niches or reproductive isolation. Subsequent speciation events from any individual species would then become increasingly more difficult as the genetic variation gets depleted. Much of the ancestral variation may still be present in the adaptive radiation but now confined to individual species. Recurrent introgressive hybridization among some of the species may slow down the depletion of genetic variation at functionally relevant genes and thus facilitate further speciation events (Grant and Grant 1992; 
Seehausen 2004). Evidence for hybridization's contribution to speciation in Lake Victoria cichlids has been found for several species (Keller, et al. 2013; Meier, et al. 2017b).

The Lake Victoria cichlid species pair Pundamilia nyererei and P. pundamilia, and other phenotypically similar species pairs, are particularly well-suited to study how selection and hybridization influence adaptation and speciation. These species coexist in full sympatry at different rocky islands in the open and offshore sectors of Lake Victoria, where they exhibit variable levels of genetic and phenotypic differentiation (Fig. 1, Seehausen, et al. 2008; Seehausen 2009). P. nyererei tends to live in deeper water than $P$. pundamilia where the light spectrum is more red-shifted, and it carries a red-shifted LWS opsin haplotype (Carleton, et al. 2005; Seehausen, et al. 2008), has red shifted color perception (Maan, et al. 2006), males have red back nuptial coloration (Fig. 1b), and females have a strong preference for red males (Maan, et al. 2004; Seehausen 2009). P. pundamilia lives in shallower water, has blue male nuptial coloration (Fig. 1b), a less red-shifted LWS opsin haplotype, blue-shifted color perception, and females prefer blue males (Seehausen and van Alphen 1998; Seehausen 2009). The two species also differ in morphology, e.g. P. pundamilia has a longer head, wider and longer lower jaws, a longer snout, deeper cheeks, smaller eyes, and less teeth but more tooth rows in the upper oral jaw than P. nyererei (van Rijssel, et al. 2018). These traits have a high heritability (Magalhaes, et al. 2009).

The Mwanza Gulf, a fjord-like extension in the south of Lake Victoria, contains many islands that also harbor red-back and blue Pundamilia that occupy similar niches as $P$. nyererei and $P$. pundamilia in the main lake (Fig. 1, Seehausen 1996; Seehausen, et al. 2008). Females have strong mating preferences for males based on color (Seehausen and van Alphen 1998; Stelkens, et al. 2008; Selz, et al. 2014). The two species show morphological differences in the same direction as $P$. nyererei and $P$. pundamilia in head length, lower jaw length, snout length and number of teeth in the upper oral jaws (van Rijssel, et al. 2018). However, the red-backed (nyererei-like) and blue (pundamilia-like) populations in the central and north-western Mwanza Gulf differ in multiple details of coloration and morphology from $P$. nyererei and P. pundamilia (Seehausen 1996; Meier, et al. 2017b; van Rijssel, et al. 2018). Some morphological traits differ between the species in opposite directions compared to $P$. pundamilia and P. nyererei. For example, $P$. sp. "pundamilia-like" has larger eyes than $P$. sp. "nyererei-like", whereas P. pundamilia has smaller eyes than P. nyererei (van Rijssel, et al. 2018). Based on microsatellite data, the two Mwanza Gulf species are genetically more similar to each other than either of them is to $P$. nyererei and $P$. pundamilia (Seehausen, et al. 2008). Females of $P$. sp. "nyererei-like" from Python Island in the Mwanza Gulf prefer conspecific males over $P$. nyererei males from outside of the Mwanza Gulf (Selz, et al. 2016), suggesting behavioral reproductive isolation between the central and northwestern Mwanza Gulf species and the species in the main lake (Fig. 1). 
We recently investigated the evolutionary history of these species with demographic modeling of $P$. pundamilia and $P$. nyererei from Makobe Island in the open lake north of the Mwanza Gulf and $P$. sp. "pundamilia-like" and P. sp. "nyererei-like" from Python Island in the central Mwanza Gulf (Fig. 1, Meier, et al. 2017b). We found that P. sp. "pundamilia-like" and P. sp. "nyererei-like" are of hybrid origin between P. pundamilia and P. nyererei (Meier, et al. 2017b). Under the best-supported model, $P$. pundamilia established first in the Mwanza Gulf, and later $P$. nyererei individuals colonized the Mwanza Gulf and admixed with the local $P$. pundamilia population. Shortly thereafter, the hybrid population speciated into a shallow living species with blue males ( $P$. sp. "pundamilia-like") and a deeper living species with red-backed males (P. sp. "nyererei-like"). Some of the allelic variation that facilitated the evolution of the new red-backed species, which colonized the deep-water niche, may have arrived from P. nyererei. In this study, we explore the sorting of admixture-derived alleles into the new species and ask to what extent phenotypic parallelism between the older and the younger species pair is reflected in genome-wide patterns of species divergence. The younger species in the Mwanza Gulf may have emerged through "re-speciation" (sensu Gilman and Behm 2011), where the new species diverge in all or a subset of the key genomic regions that the older species pair had already experienced divergence in. Alternatively, the evolution of these species may resemble a scenario of "hybrid parallel speciation", where the new species parallel the older ones phenotypically and in some of the divergent genomic regions, but additionally diverge in many other regions in the genome that are not divergent in the older species pair.

Candidate genes that may have facilitated Pundamilia speciation include the red-sensitive (LWS) opsin gene, which is involved in adaptation to deep versus shallow water light environments (Seehausen, et al. 2008), the blue-sensitive opsin gene SWS2a (Seehausen, et al. 2008), genes mediating red versus blue male nuptial coloration (Magalhaes and Seehausen 2010), genes contributing to morphological (Magalhaes, et al. 2009; van Rijssel, et al. 2018) or metabolic differences involved in diet shifts (Seehausen, et al. 1998), or genes conferring female preference for red or blue male nuptial coloration (Svensson, et al. 2017). The hybrid parallel speciation scenario, where the second speciation event is facilitated by introgression of $P$. nyererei genes into a local population of $P$. pundamilia (Meier, et al. 2017b), predicts that genomic regions carrying such genes should be differentiated in the original species pair and be among the most strongly differentiated genes in the younger pair. However, there should also be many other differentiated regions between the younger species that are not shared with the original older species pair. These other regions would have become differentiated during the new speciation process. In contrast, "re-speciation" in the sense of restoration of the original species differences after massive hybridization would imply that regions of strong genomic differentiation in 
the young species pair are a subset of the genomic regions of high differentiation in the original species pair.

Here, we use whole-genome re-sequencing data from the sympatric Pundamilia species pairs at Makobe Island (the older species pair) and at Python Island (the very recent species pair) (Meier, et al. 2017b) to characterize the genomic patterns and potential parallelism of divergence in the species pairs, assess the role of admixture-derived variation, and distinguish between the re-speciation and the hybrid parallel speciation scenarios. We find that many of the genomic regions that are most strongly differentiated between the older $P$. nyererei and $P$. pundamilia are also differentiated between the younger $P$. sp. "nyererei-like" and $P$. sp. "pundamilia-like" and mostly show high allele sharing between older and younger species of the same color type. However, two thirds of the high differentiation regions in the young species pair constitute novel candidate targets of divergent selection in this pair, as they show no signature of selection in the original species pair, consistent with hybrid parallel speciation.

\section{Results}

\section{Nucleotide diversity and divergence}

We used whole-genome re-sequencing data of four individuals each of $P$. nyererei and $P$. pundamilia from Makobe Island (the older original species) and of $P$. sp. "nyererei-like" and P. sp. "pundamilialike" from Python Island (the younger species in the Mwanza Gulf). By mapping the reads to the anchored $P$. nyererei reference genome (Feulner, et al. 2018), we obtained 630 million base pairs (Mbp) including 5,199,846 bi-allelic SNPs sequenced in at least half of the individuals with a minimum of 10 reads each (mean depth of coverage is 16-29 reads, Table S1). The mean missing data proportion per site was 3.1\%. The mean proportion of bi-allelic SNPs among all sites in the dataset combining all populations was $0.44 \%$ (excluding sites with missing data, Table S1) and not much lower in single species datasets $\left(0.36-0.41 \%\right.$, Table S1). The mean weighted $\mathrm{F}_{S T}$ was 0.108 between $P$. pundamilia and P. nyererei at Makobe, 0.053 between P. sp. "pundamilia-like" and P. sp. "nyererei-like" at Python (Fig. 1) based on sites without missing data (on average 2.1 million SNPs). The vast majority of SNPs were shared across multiple or all species. Of the SNPs with maximum one individual missing per species and a minor allele count of three across all four species, only $1 \%$ of the variants were unique to $P$. pundamilia Makobe, $0.31 \%$ to $P$. nyererei Makobe, $0.36 \%$ to $P$. sp. "pundamilia-like" Python, and $0.24 \%$ to $P$. sp. "nyererei-like" at Python Island. $4.2 \%$ of these variants were only found in the species at Makobe and $4.4 \%$ only in the species at Python Island. 


\section{One third of the high differentiation regions are shared between species pairs}

To assess if the genomic differentiation between P. sp. "nyererei-like" and P. sp. "pundamilia-like" paralleled that between $P$. nyererei and $P$. pundamilia, we identified highly differentiated genomic regions for Python and Makobe Island separately, using a Hidden Markov Model (HMM, Baum and Petrie 1966) approach. For each species pair, we computed a null model expectation for genome-wide differentiation $\left(F_{S T}\right)$ from simulations under the best demographic model in Meier, et al. (2017b) (Fig S1). Based on these simulated null distributions we computed z-scores (normalized p-values) for our observed $\mathrm{F}_{\mathrm{ST}}$ values averaged across 15 SNPs. Given that the parameters of the demographic model were estimated using a dataset that contained both neutrally and non-neutrally evolving genomic regions (Meier et al., 2017b), the null distributions are not purely neutral and thus outlier detection using this null model is highly conservative. An HMM with two hidden states corresponding to "normal" and "high differentiation" was run over the z-scores. Of the 137,550 15-SNP windows in the Makobe dataset and 112,478 15-SNP windows in the Python dataset, 5.4\% and 5.9\% respectively had a z-score above 1.65 (corresponding to a one-sided p-value of 0.05 ), and $7.8 \%$ and $7.1 \%$ were assigned to the high differentiation state in the Makobe and Python datasets, respectively (Table 1).

The mean $\mathrm{F}_{\mathrm{ST}}$ of windows assigned to the highly differentiated state was 4.5 and 6.7 times as high as the mean of the other windows at Makobe Island (0.296 vs 0.066 ) and Python Island (0.207 vs 0.031 ), respectively (Table 1, Fig. S1). When highly divergent regions were excluded, the genome-wide mean $F_{S T}$ from SNPs pruned for high linkage $\left(r^{2}>0.5\right)$ was 0.031 with a $99 \%$ bootstrap confidence interval $(\mathrm{Cl})$ of 0.030-0.032 for the young species pair at Python Island and $0.058(99 \% \mathrm{Cl}=0.056-0.059)$ for the older species pair at Makobe Island, closely resembling previously published $F_{S T}$ estimates from microsatellites (Seehausen, et al. 2008). This suggests that both pairs of sympatric species are experiencing some genome-wide reproductive isolation also outside the high divergence regions.

We combined adjacent high differentiation windows to highly divergent genomic regions (HDRs). This approach resulted in 346 Makobe HDRs and 365 Python HDRs, respectively (Table 1). Makobe HDRs were larger than Python HDRs (on average 268 vs 196 kb, two-sided Mann Whitney U-test, pvalue $=0.02$, Table 1, Fig. S2). By extracting the parts of the HDRs that overlap between Makobe and Python Island, we obtained 120 shared HDRs (SHDRs) that were widely scattered across the genome (Fig. 2, blue vertical lines). Of the 346 Makobe HDRs, 103 overlapped with at least one Python HDR (29.8\%), indicating that only a subset of the highly divergent regions of the original species pair is also divergent between the younger species at Python Island. Of the Python HDRs, a very similar fraction of $28.8 \%$ (105/365 HDRs) overlapped with at least one Makobe HDR, indicating that many unique genomic regions show signatures of divergent selection in the younger species pair that were not 
divergent between the older species. The proportion of HDRs shared between both species pairs is robust to different approaches and window sizes used to identify HDRs (Table S2). All shared HDRs together covered $21.1 \mathrm{Mb}$, which is $22.6 \%$ and $29.3 \%$ of the HDR lengths at Makobe and Python Islands, respectively. Both the length distribution of HDRs (Fig. S2a,b) and that of SHDRs was strongly righttailed (Fig. S2c), the latter with mean $176 \mathrm{~kb}$, median $91 \mathrm{~kb}$, and a maximum length of 1,5 Mbp. The overlap of HDRs from Python and Makobe is much larger than expected in a block-permuted dataset (Fig. S3).

We computed $\mathrm{F}_{\text {ST }}$ estimates for both species pairs in non-overlapping $20 \mathrm{~kb}$ windows to compare $\mathrm{F}_{S T}$ values of the same genomic regions between the two pairs. The HDRs unique to one species pair did in the other species pair not show higher $\mathrm{F}_{S T}$ values than the genome-wide background (Fig. S4). The FsT $_{\text {ST }}$ estimates for both species pairs were slightly and significantly higher in shared HDRs than in HDRs unique to either of the species pairs (Fig. S4), suggesting that many of the most strongly divergent regions between $P$. nyererei and $P$. pundamilia were also targeted by divergent selection in the younger species pair at Python Island.

\section{Highly divergent genomic regions show signatures of selection in multiple statistics}

We further characterized highly divergent genomic regions by computing additional statistics for selection, nucleotide diversity, recombination rate, and introgression in windows of $20 \mathrm{~kb}$. We obtained 32,864 $20 \mathrm{~kb}$ windows with an average of 17,508 covered sites (median: 18,286) and 130 SNPs. For Makobe and Python Island separately, we compared $20 \mathrm{~kb}$ windows overlapping with HDRs (HDR windows) with windows overlapping with shared HDRs (shared HDR windows), and with windows not overlapping with any HDRs representing the genome-wide background (nonHDR windows).

We tested whether HDRs were enriched for signatures of selection using two distinct "extended haplotype length" (EHL). Both tests are based on the prediction that in a selective sweep, a variant rises to high frequency so rapidly that linkage disequilibrium with neighboring polymorphisms is not disrupted by recombination, giving rise to haplotypes of extended length. The first of these tests that we computed is the Cross Population Extended Haplotype Homozygosity statistic (XP-EHH, Sabeti, et al. 2007) which compares the haplotype lengths between the sympatric sister species. A selective sweep in one of the species would lead to longer haplotypes compared to the other species (Sabeti, et al. 2007). In addition, we computed the integrated haplotype score which compares the lengths of different haplotypes within a population (iHS, Voight, et al. 2006). This test has most power during an ongoing sweep, when the haplotype under positive selection is expected to be much longer than the other haplotypes but is not fixed yet. We used absolute iHS as we did not have information on the derived or ancestral state of the alleles, and absolute XP-EHH as we did not distinguish in which of the 
diverging species in a pair selection acted. We found that compared to regions that are not highly differentiated (nonHDRs), HDRs unique to the Makobe species pair had generally higher XP-EHH and iHS values computed for the Makobe species but not for the Python species. Similarly, compared to nonHDRs, HDRs unique to the Python species pair had on average higher XP-EHH and iHS values computed for the Python species but not for the Makobe species (Fig. S5).

Of the genomic regions that were highly differentiated either only in the older or only in the younger species pair, $61 \%$ and $49 \%$, respectively, showed significant extended haplotype length statistics (significant iHS in at least one species and/or significant XP-EHH) in at least $20 \%$ of the spanned windows (Fig. 4c and S10, excluding HDRs without EHL information). The fraction of HDRs that are EHL outlier HDRs is 3.0 (Makobe) or 2.5 (Python) times as high as the average fraction obtained from blockpermuted null distributions (Fig. S10). Shared HDRs were even more frequently outliers for EHL statistics (3.3x and 2.6x enrichment for Makobe and Python EHL signatures, respectively, Fig. S6). However, HDRs unique to the Makobe species were not enriched for Python EHL outliers, and HDRs unique to the Python species pair were not enriched for Makobe EHL outliers (Fig. S6).

As a further test of divergent selection, we computed the difference in nucleotide diversity $(\Delta \mathrm{pi})$ between the species in windows of $20 \mathrm{~kb}$. A high absolute $\Delta \pi$ indicates that a selective sweep reduced the nucleotide diversity in one population. We considered windows with a $\Delta \pi$ value above the $90^{\text {th }}$ quantile as $\Delta \pi$ outlier windows providing additional evidence of divergent selection. Makobe HDRs were enriched for Makobe $\Delta \pi$ outlier windows and Python HDRs were enriched for Python $\Delta \pi$ outlier windows supporting that HDRs were formed by divergent selection (Fig. 4c, S10). In contrast, HDRs unique to one species pair did not show enrichment for high $\Delta \pi$ in the other species pair (Fig. 4c, S10), supporting absence of divergent selection in the other species pair.

As yet a further test, we explored signatures of selection using a measure of absolute sequence divergence. In contrast to relative measures of divergence such as $F_{S T}$, which compare genetic differentiation between populations to genetic variation within populations, absolute measures of divergence are less affected by forces decreasing within-population variation such as background selection (Nachman and Payseur 2012; Cruickshank and Hahn 2014). Nei's measure of absolute divergence $\left(d_{X Y}\right)$ calculated in windows of $20 \mathrm{~kb}$ was highly correlated with within-species diversity (Fig. S7, Pearson's correlation $\left(r^{2}\right)$ between $d_{x y}$ and the average of the two $\pi$ estimates is 0.81 for the older species pair at Makobe and 0.92 for the younger species pair at Python). This is in line with the prediction that for comparisons of very recently diverged taxa, $\mathrm{d}_{\mathrm{Xy}}$ mostly correlates with mutation rates and levels of ancestral variation (Nachman and Payseur 2012; Cruickshank and Hahn 2014; Riesch, et al. 2017). We identified outlier windows where high $d_{x y}$ values are unlikely to be due to 
increased mutation rate: windows with both $d_{X Y}$ and $d_{X Y} / \pi$ values higher than 1.5 times the interquartile range (IQ) above the third quartile (Q3). Using this two-pronged approach, we identified 134 and $83 d_{X y}$ outlier windows for the older and the younger species pair, respectively (Fig. 2). Of these windows with high $d_{x y}$ between the older species, $83 \%$ were distributed across 63 different HDRs, of which 17 were shared HDRs. Similarly, $84 \%$ of the windows with high $d_{x y}$ in the younger species pair were located in 46 HDRs, of which 14 were shared with the older species pair. HDRs unique to either one of the species pairs were strongly enriched for outlier windows of $d_{X Y}$ in the respective species pair compared to block-permuted null expectations ( $4 x$ for the older species pair, $6 x$ for the younger species pair, Fig. 4, S8). However, HDRs unique to one species pair did not show enrichment for $d_{x y}$ outlier windows (Fig. 4) in the other species pair, confirming that these regions were only under divergent selection in the focal species pair. Shared HDRs were most strongly enriched for $d_{X y}$ outliers with $6 x$ and $9 x$ enrichment compared to the mean of block-permuted shared HDRs for Makobe and Python $d_{X Y}$ outliers, respectively (Fig. 4 and S9).

Finally, as yet one more signature of selection that also captures regions with ongoing and completed selective sweeps, we computed Tajima's D in $20 \mathrm{~kb}$ windows. After a sweep, the beneficial haplotype will reach high prevalence and new mutations will only arise slowly and initially be at low frequency. This excess of rare alleles renders Tajima's D values negative in sweep regions. However, we caution that in the very young species pair at Python Island, new mutations that arose after the onset of speciation are expected to be very few if any and Tajima's D may not convey much of a selection signal. If at least $20 \%$ of the windows spanned by the HDR had strongly negative Tajima's D values below the fifth quantile for one or both species, we considered the HDR to be a Tajima's D outlier HDR (Fig. 4c, S10). As expected, we found a strong enrichment of Tajima's D outlier HDRs in the older original species pair (3x) but only a weak enrichment in the young species pair (Fig. 4c, S10). HDRs unique to the original species pair were also slightly enriched for strongly negative Tajima's D in the younger species pair, which is likely a legacy of past selection in the original species pair (Fig. 4c, S10). This might generally be a problem in cases of recent divergent events within populations of admixed ancestry and warrants caution in the interpretation.

Of the HDRs unique to the older or to the younger species pair with information for all five statistics of selection (XP-EHH, iHS, $d_{X Y}, \Delta \pi$, Tajima's $D$ ) in addition to $F_{S T}, 72 \%$ or $64 \%$, respectively, were significant for at least one other selection statistic. The percentage of HDRs unique to one species pair showing additional evidence for selection in that species pair was much higher than expected from blockpermuted null distributions (Fig. 4a-b, Fig. S8). The shared HDRs were even more strongly enriched for additional evidence of selection (Fig. 4a-b, Fig. S8). Of the 120 shared HDRs, 100 showed additional 
significant selection statistics in the original species pair at Makobe Island and 95 in the young species pair at Python Island (113 in at least one of the species pairs). The different selection statistics thus strongly support the action of divergent selection in driving the high differentiation in HDRs. In contrast, HDRs unique to one species pair are not enriched for significant selection statistics in the other species pair, as shown by block-permutation (Fig. 4a-b). This indicates that HDRs unique to one species pair are indeed not under divergent selection in the other species pair.

\section{Genomic regions under divergent selection are associated with low recombination rates}

We tested if highly differentiated genomic regions were associated with lower than expected recombination rates. Regions under strong divergent selection may be associated with low recombination because selection reduces effective gene flow and recombination between co-adapted alleles (Rieseberg 2001; Butlin 2005; Hoffmann and Rieseberg 2008; Bürger and Akerman 2011; Yeaman and Whitlock 2011; Nachman and Payseur 2012). However, similar patterns of strong differentiation in low recombination regions can be caused by purifying selection against recurrent deleterious mutations (termed background selection, Charlesworth, et al. 1993; Nachman and Payseur 2012) or against maladaptive introgressed alleles (Martin and Jiggins 2017). In the absence of gene flow, background selection is likely to be the major driver of the commonly observed association between high divergence and low recombination rates (Nachman and Payseur 2012; Renaut, et al. 2013; Cruickshank and Hahn 2014; Burri, et al. 2015). However, in cases of recent and sympatric divergence with ongoing gene flow, such as the young Pundamilia species pair at Python Island, background selection is unlikely to cause regions of low recombination to diverge between the species (see also Cruickshank and Hahn 2014; Burri 2017).

We found that the average recombination rates were lower in HDRs compared to the rest of the genome (Fig. 3). Shared HDRs were even more strongly associated with low average recombination rates (Fig. 3). This finding was consistent if we restricted the analyses to HDRs with additional evidence for divergent selection (Fig. S9) suggesting that divergent selection rather than background selection is driving the association. We found a genome-wide negative association between recombination rate and multiple statistics of selection ( $\mathrm{iHS}, \mathrm{XP}-\mathrm{EHH}, \Delta \mathrm{pi}$ ), which are less likely to be affected by background selection than $\mathrm{F}_{\mathrm{ST}}$ (Fig. S10). Outlier windows of high absolute divergence $\left(\mathrm{d}_{\mathrm{xy}}\right)$ are unlikely to be caused by background selection and also had a reduced mean recombination rate compared to the rest of the windows (1.99 vs $2.32 \mathrm{cM} / \mathrm{Mb}$ for the older species pair and $1.91 \mathrm{vs} 2.32 \mathrm{cM} / \mathrm{Mb}$ in the younger pair).

In addition, if background selection was driving the similarity in genomic differentiation landscapes between species pairs (e.g. Renaut, et al. 2013; Burri, et al. 2015) and contributing to shared HDRs in 
low recombination regions, we would expect high correlation of $F_{S T}$ window estimates between the species pairs. However, we found low overall correlation of genetic differentiation patterns between the species pairs even though the recombination map is likely highly similar (Fig. S11). Using the RAD data from Meier et al., (2017b), we computed $F_{S T}$ estimates between the older species at Makobe Island, the younger species at Python Island and at another island (Kissenda Island, Fig. 1), and between each species at Python Island and an allopatric Pundamilia population at a fourth island (Luanso Island in the South of the Mwanza Gulf, Fig.1). We tested if $20 \mathrm{~kb}$ windows that are highly differentiated between the older species pair at Makobe Island (mean $\mathrm{F}_{\mathrm{ST}}>0.2$ ) are also more strongly differentiated between allopatric species that are unlikely to experience parallel selection pressures. We found that in these windows, differentiation of either species from Python Island against the allopatric Luanso population was not higher than expected from block-permutation null distributions (Fig. S10c). In contrast, windows that were highly differentiated between the older species at Makobe Island also showed elevated differentiation between the sympatric younger species at both islands with sympatric species pairs (Python and Kissenda Island, Fig. S11c, permutation test: $p<0.001$ ). This provides additional evidence that parallel divergent selection and not background selection drives the elevated differentiation in the sympatric species pairs.

\section{Parallel selection on same alleles in the old and young species pairs}

To test if species with the same male nuptial color syndrome (red-back vs blue) share more alleles in HDRs than species with different male color syndromes, we computed $f_{d}$ (Martin, et al. 2015), a measure of excess allele sharing in windows of $20 \mathrm{~kb}$. We found that $f_{d}$ among same-color species is much higher in shared HDR windows than in other windows, indicating high allele sharing between same-color species. Mean $\mathrm{f}_{\mathrm{d}}$ yer, measuring excess allele sharing between $P$. nyererei with $P$. $\mathrm{sp}$. "nyererei-like" relative to $P$. sp. "pundamilia-like", was 0.27 in shared HDRs, but only 0.12 in the rest of the genome. Excess allele sharing between P. pundamilia and P. sp. "pundamilia-like" compared to $P$. sp. "nyererei-like" ( $f_{d}$ pund) was on average 0.18 in shared HDRs but only 0.08 outside of shared HDRs. Shared HDRs, were strongly enriched for top $10 \% \mathrm{f}_{\mathrm{d}}$ nyer and top $10 \% \mathrm{f}_{\mathrm{d}}$ pund windows (Fig. $4 \mathrm{~d}$ e), which indicates that divergent selection in these regions in the younger species pair had acted on admixture-derived alleles. Of the shared HDRs with available estimates, about $60 \%$ overlapped with at least one top $10 \% \mathrm{f}_{\mathrm{d}}$ nyer or $\mathrm{f}_{\mathrm{d}}$ pund window (Fig. $4 \mathrm{~d}$-e).

As another way to identify genomic windows with parallel allele frequency differences between the sympatric species at Makobe and Python Island, we assessed the most likely species tree topology in non-overlapping $20 \mathrm{~kb}$ windows using TWISST (Martin and Van Belleghem 2017). Overall, the "geography topology" which groups the species by island (Python or Makobe Island, respectively) is 
best supported (Fig. S12). However, in shared HDRs, the "color topology", where species are grouped by male nuptial color syndrome $((P$. nyererei $+P$. sp. "nyererei-like"), $(P$. pundamilia $+P$. sp. "pundamilia-like")) is more frequent than the "geography topology", consistent with parallel selection on shared genetic variation (Fig. S12). Shared HDRs had higher weights for the color topology and lower weights for the geography and the random topology compared to the genome-wide average (permutation test, $\mathrm{p}<0.001$ for all three topologies). We considered windows where the "color topology" weight exceeds 66\% (twice as high as both other possible topologies together) as "color topology windows". As the sharing of variation is likely due to the admixed ancestry of the younger species, "color topology windows" indicate regions where the admixture variation was sorted between the species in the younger species pair under selection pressures paralleling those experienced by the species in the older species pair. The proportion of shared HDRs containing such a "color topology window" was much higher than expected from block-permuted null distributions ( $34 \%, p<0.01$, Fig. $4 f)$.

Shared HDRs with at least one "color topology window" were considered candidate regions of parallel selection on admixture-derived genetic variation (Fig. 4f). We identified 41 such candidate regions containing 71 "color topology windows" (Fig. 2). These regions overlapped with 182 known genes (Table S3, see also a visualization of the SHDRs with gene info on Dryad). These genes were spread across 25 of the 41 candidate regions of parallel selection. Among these candidate genes 27 genes are involved in visual perception or nervous system development and may thus contribute to divergent adaptation to different light regimes at different water depths and to behavioral reproductive isolation based on male nuptial coloration. Four genes are associated with pigmentation and might confer differences in male nuptial coloration. Three genes are opsin genes known to be under divergent selection between the species and are described in more detail in the next section. Twenty-three other candidate genes are associated with carbohydrate, lipid and folic acid metabolism. They may be involved in diet shifts between the Pundamilia species (Bouton, et al. 1997). Fifteen further candidate genes are involved in immunity and may thus confer adaptation to divergent parasite pressures at different water depth (Maan, et al. 2008). Eight candidate genes are associated with limb, bone and cartilage development and may contribute to morphological differences between the species (Seehausen, et al. 1998; Magalhaes, et al. 2009; van Rijssel, et al. 2018). Fifteen other genes are associated with divergence in gene expression (transcription factors). Functional validation in further studies is required for most of these candidate genes of parallel divergence, including 36 genes currently lacking functional annotation, three candidate genes with a wide array of functions (Table S3), and the 16 candidate regions currently lacking known genes. 


\section{Divergence in the LWS opsin gene region}

The long wavelength sensitive ( $L W S$ ) opsin gene is known to be importantly involved in adaptation to the different ambient light spectra at different water depths and is also correlated with male nuptial coloration and with female mate choice based on male nuptial coloration in Pundamilia cichlids (Carleton, et al. 2005; Maan, et al. 2006; Seehausen, et al. 2008), and has recently been shown to directly determine variation in mating preferences in medaka (Kamijo, et al. 2018). Seehausen, et al. (2008) showed that $P$. pundamilia and $P$. sp. "pundamilia-like" predominantly have the $\mathrm{P}$ haplotype, whereas $P$. nyererei and $P$. sp. "nyererei-like" are almost fixed for the $\mathrm{H}$ haplotype, whose protein light absorption spectrum is red-shifted by $15 \mathrm{~nm}$ relative to the $\mathrm{P}$ haplotype. This led Meier, et al. (2017b) to hypothesize that the LWS opsin gene may represent a candidate gene for introgression from $P$. nyererei into Python Pundamilia that would have facilitated the evolution of $P$. sp. "nyererei-like" through parallel speciation.

The LWS opsin gene is located on linkage group 13 in the anchored P. nyererei reference genome (Fig. 5) corresponding to scaffold 177 of the original genome assembly by Brawand, et al. (2014). Here, we found that the genomic region around this gene is highly differentiated between the sympatric species both at Makobe and at Python Island. The $\mathrm{F}_{\mathrm{ST}}$ estimate of the 15-SNP window overlapping with the LWS gene was 0.84 (99.992 ${ }^{\text {th }}$ quantile) for the younger species pair, and $0.58\left(99.434^{\text {th }}\right.$ quantile) for the older species pair. The region of overlap between Makobe and Python HDRs spanned five genes including, besides $L W S$ also the blue-sensitive opsin gene (SWS-2A) and the violet-sensitive opsin gene (SWS-2B, Fig. 5). Three $20 \mathrm{~kb}$ windows overlapped with this region. For two of the three $20 \mathrm{~kb}$ windows spanning the region, $f_{d}$ nyer values were available and estimated to be very high $(0.80$ and 0.22 , corresponding to the $99.7^{\text {th }}$ and the $76.4^{\text {th }}$ quantile) indicating high excess allele sharing between the two red-backed species. The third window contained too few informative SNPs to estimate $f_{d}$ nyer but had a high $f_{d}$ pund estimate $\left(0.18,80^{\text {th }}\right.$ quantile) indicating excess allele sharing between the two species with blue male nuptial coloration. The $20 \mathrm{~kb}$ window overlapping with the LWS gene shows strong clustering of the species by male coloration. The "color topology" has a weight of 0.84 , whereas the otherwise predominant "geography topology" has a weight of only 0.06 in this window.

Six sites in the LWS gene showed parallel differences in allele frequency between the older species at Makobe Island and the younger species at Python Island. The blue (SWS-2A) and violet vision (SWS-2B) opsin genes also each contained multiple sites with large parallel allele frequency shifts between the species at both islands, whereas most sites in intergenic regions were highly differentiated either only between the species at one island or at both islands but with non-parallel allele frequency shifts (Fig. $5)$. 


\section{Discussion}

In this study, we explore genomic patterns of differentiation in a cichlid species complex that we previously showed to consist of a younger and an older pair of sympatric sister species, each with one species with blue and the other with red-back male nuptial coloration. The younger pair evolved outside the distribution range of the older red species after hybridization between the latter and an allopatric population of the older blue species. We show that the genomic landscape of differentiation in both species pairs is heterogeneous with highly differentiated genomic regions spread across the entire genome even in the very young species pair. A third of the genomic regions that are most strongly differentiated between the older species at Makobe Island are also strongly differentiated between the younger species at Python Island. However, two thirds of the genomic regions that are differentiated between the young species are not differentiated between the older species at all, consistent with an independent speciation event rather than "re-speciation". In the following, we will discuss each of these results in more detail.

\section{Widespread genomic regions of high interspecific differentiation}

We find highly heterogeneous patterns of genome-wide differentiation between the species. Both at Makobe and at Python Island, almost $8 \%$ of all 15-SNP windows were assigned to the highly differentiated state, and these were clustered into more than 300 highly differentiated genomic regions in each species pair (Table 1). In the younger species pair at Python Island, we detected slightly more but smaller differentiated genomic regions than in the much older original species pair at Makobe Island. Some of these HDRs may be false positives, but we find a strong enrichment for additional selection statistics indicating that differentiation in most of these regions was driven by divergent selection. The large number of highly differentiated regions in the species at Python Island and significant genome-wide divergence even after exclusion of HDRs may seem surprising given that the species are estimated to be very young (probably <250 generations, Meier, et al. 2017b). Gene flow is likely ongoing as suggested by the quite high estimates of interspecific gene flow (Seehausen, et al. 2008; Meier, et al. 2017b) and observations of intermediate phenotypes in nature (Seehausen 1997; Seehausen, et al. 2008; Seehausen 2009).

In young incipient species that diverge in the face of gene flow and are not fully isolated yet, restriction of gene flow is thought to be initially confined to very few genomic regions that experience strong divergent selection, a prediction that finds some support in other study systems (Kronforst, et al. 2013; Nadeau, et al. 2013; Marques, et al. 2016; Marques, et al. 2017). However, here we showed that when large genetic variation due to hybrid ancestry is present at functionally relevant genes, extensive 
genetic differentiation at many and genomically widespread loci may characterize even very young cases of sympatric speciation.

Divergent selection acting simultaneously on many regions across the genome may eventually lead to genome-wide reductions in gene flow (Barton 1983; Feder, et al. 2012a; Feder, et al. 2012b; Flaxman, et al. 2012; Feder, et al. 2014). Genome-wide reproductive isolation in ecological speciation with gene flow may result from "genomic congealing" (Feder, et al. 2014; Flaxman, et al. 2014). In this model, during a period of divergent selection in the face of gene flow, low levels of linkage disequilibrium (LD) between many loci under divergent selection slowly build up, until positive feedback between LD and selection increases the effectiveness of selection, leading to a sudden cessation of gene flow (Feder, et al. 2014; Flaxman, et al. 2014).

Whereas models of genomic congealing require a very long phase of LD building preceding the sudden emergence of reproductive isolation, we find that despite the very young age of the sympatric species at Python Island, they already show genome-wide differentiation. This indicates that the slow phase involving the building of LD may not be needed if divergent selection acts on a hybrid population with segregating ancestry tracts containing multiple linked beneficial loci that evolved in response to selection in the source species. Hybrid populations will thus display large variation for the traits differing between the source species and linkage between some co-adapted alleles is already present. This may allow a hybrid population to quickly respond to divergent selection in conditions resembling those under which the source species had diverged (here e.g. to deeper water, red-shifted light spectra and female preferences for red male coloration under these conditions versus more shallow water, less red-shifted light and female preference for blue male coloration). Hybridization may also cause allele combinations that lead to transgressive traits, novel trait combinations, or intrinsic and environment-mediated incompatibilities (reviewed in Abbott, et al. 2013). Introgressive hybridization may thus importantly enhance the genetic variation allowing a population to speciate rapidly in response to divergent selection.

Some genomic regions of high differentiation are very large, the largest being over $2 \mathrm{Mb}$, and we find that on average HDRs fall into regions of relatively lower recombination rate than the rest of the genome (Fig. 3). When species diverge in the face of gene flow, regions of high differentiation are often associated with regions of low recombination (Butlin 2005; Turner, et al. 2005; Hoffmann and Rieseberg 2008; Noor and Bennett 2009; Renaut, et al. 2013; Roesti, et al. 2013; Marques, et al. 2016; Samuk, et al. 2017). Theoretical models predict that regions of restricted recombination may facilitate species formation or persistence despite gene flow by protecting linkage disequilibrium among alleles conferring adaptation or barriers to gene flow (Rieseberg 2001; Bürger and Akerman 2011; Yeaman 
and Whitlock 2011). This may be particularly important in cases of hybrid parallel speciation, because co-adapted alleles may remain linked in low recombination regions in the hybrid population and thus more quickly diverge again under parallel selection pressures. Background selection is also expected to generate high $\mathrm{F}_{S T}$ values in regions of low recombination as it locally reduces the nucleotide diversity within species and thus inflates the relative divergence between the species (Charlesworth, et al. 1993; Noor and Bennett 2009; Nachman and Payseur 2012; Cruickshank and Hahn 2014). However, this would be unexpected when incipient species are fully sympatric and exchange genes as is the case in the young species pairs we studied here (see also Cruickshank and Hahn 2014; Burri, 2017). Indeed, we find overall low correlation between $F_{S T}$ estimates for the different species pairs (Fig. S11) and strong additional signatures of selection in highly divergent regions (Fig. 4), making a scenario where background selection is the main driver of divergence unlikely. Our study thus adds to the growing body of literature demonstrating an important role of variation in recombination rates in speciation with gene flow.

\section{Evidence for hybrid parallel speciation}

More than $70 \%$ of the HDRs detected in the younger species pair at Python Island show no signatures of selection in the older species pair, suggesting that the younger species are not differentiated simply in a subset of the genomic differences that characterize the older species pair. It rather suggests that evolution of the younger species pair at Python Island involved divergence in many novel genomic regions, too. Given the very young age of this species pair, and the low proportions of alleles not shared with the older species pair, it is unlikely that de novo mutations underlie these novel HDRs. The alleles under divergent selection only in the younger pair may have been segregating neutrally in the older species pair or they may have been introduced into the younger species through introgression from a third species in the Mwanza Gulf.

Overall, there is low correlation between the differentiation landscapes of the two species pairs (Fig. S11), consistent with substantial independent evolution of these pairs. In general, the findings suggest that $P$. sp. "nyererei-like" and $P$. sp. "pundamilia-like" of Python Island and the north-western Mwanza Gulf do not merely represent re-assembled genomes of the original $P$. nyererei and $P$. pundamilia (respeciation scenario sensu Gilman and Behm, 2011). Instead, our data support the prediction of a hybrid parallel speciation hypothesis that the younger species of the Mwanza Gulf diverged largely independently from the older species pair outside of the Mwanza Gulf, whereby parallel selection targeted admixture-derive haplotypic variation in some genomic regions. The finding that shared HDRs have on average higher mean $\mathrm{F}_{\mathrm{ST}}$ values (Fig. S4) and a stronger enrichment of other selection statistics (Fig. 4, S9, and S10) than HDRs unique to either one of the species pairs, suggests that the haplotypes 
under strongest divergent selection in the original species pair were importantly involved in the divergence of the younger species pair.

\section{Modest levels of genomic parallelism despite strong phenotypic parallelism}

Even though the overlap of highly differentiated genomic regions detected in the species pairs of Python and Makobe Islands is higher than expected from random permutations (Fig. S3), less than a third of the HDRs in each species pair are shared between the two species pairs. This amount of genomic parallelism is unexpectedly modest given the extreme recency of the younger species pair (likely $<250$ generations, Meier, et al. 2017b). The probability of gene reuse under parallel selection pressures is expected to be very high when selection acts on shared standing variation (around $80 \%$, Conte, et al. 2012; which may be slightly different at the level of genomic regions Roda, et al. 2013). Due to the recent hybrid origin of both species sampled at Python Island and ongoing gene flow between all four species, as well as other additional cichlid species, the availability of genetic variation is unlikely to be a major constraint on parallel evolution in Pundamilia cichlids.

Modest parallelism at the genomic level despite strong phenotypic parallelism has also been found in many other young species/ecotype pairs, such as wave-swept and crab-predated Littorina ecotypes (Ravinet, et al. 2016), or ecomorphs of Timema stick insects (Soria-Carrasco, et al. 2014). Striking phenotypic parallelism despite modest levels of genomic parallelism may have multiple explanations and distinguishing among them is challenging. These explanations include: 1) The phenotypes may have a quantitative or complex genetic basis with multiple genes in the same pathway that can be tuned by selection with similar phenotypic output (Hoekstra and Nachman 2003; Pritchard, et al. 2010; Boyle, et al. 2017). 2) The few shared HDRs may underlie phenotypic parallelism, while the additional HDRs unique to each species pair may be under non-parallel divergent selection pressures, e.g. due to different parasite regimes, diets or water clarity. 3) The observed phenotypic parallelism may be mainly due to phenotypic plasticity (Lande 2009; Pfennig, et al. 2010). However, phenotypic plasticity can be ruled out as a cause of many species differences in Pundamilia (Seehausen 2009). There is evidence that the genetic basis underlying female mate choice and male nuptial coloration in the young Pundamilia species pair of Python Island is fairly simple and based on only a small number of physically unlinked Mendelian factors (Haesler and Seehausen 2005; Magalhaes and Seehausen 2010; Svensson, et al. 2017) that may consist of single genes or multiple linked genes. For most other traits that differ between the species at Makobe and at Python Island, the complexity of the genetic basis is unknown.

Some of the lack of genomic parallelism may be attributed to differences in the detection probabilities of signatures of selection in the older and younger species pair. The lower overall $\mathrm{F}_{\mathrm{ST}}$ in the younger species pair may facilitate the detection of high $\mathrm{F}_{S T}$ outliers compared to the older species pair. 
Additionally, sorting of divergent ancestry blocks containing one or multiple sites under divergent selection in the younger species may generate a strong signal. If only a few adaptive haplotypes were present in the admixed ancestry of the young species, the signal may resemble a hard sweep. The detection of such regions in the younger species pair may thus be easier than the detection of regions under long-term divergent selection (repeated soft sweeps) in the older species pair. On the other hand, sweeps may still be ongoing in the younger species pair and thus harder to detect. However, the different selection statistics we used in addition to $F_{S T}$ have varied advantages and limitations in detecting different kinds of sweeps. As an example, because iHS is most sensitive to ongoing sweeps (Voight et al., 2006), it may have more power to detect recent sweeps in the younger species pair, whereas XP-EHH, which is most sensitive when the selected allele is near fixation (Sabeti et al., 2007), may have more power in the older species pair. The combination of these different statistics in our analysis should thus account for differences in selection signals in the two species pairs. Together, the array of selection statistics we used strongly support the presence of divergent selection in HDRs unique to one species pair and the absence of divergent selection in the same regions in the other species pair (Fig. 4a-b).

\section{Shared highly differentiated genomic regions contain candidates for parallel divergent selection}

Genomic regions that are highly differentiated between both species pairs are enriched for regions with high allele sharing between both red-backed species (high $\mathrm{f}_{\mathrm{d}}$ nyer, Fig. $4 \mathrm{~d}$ ) and (to a lesser extent) high allele sharing between both blue species (high $f_{d}$ pund, Fig. 4e). In many SHDRs, the species group more strongly by male nuptial coloration than by sampling location, against the genome-wide phylogenetic signatures (Fig. 4f). The excess allele sharing between more distantly related species of similar phenotype in these genomic regions could be due to 1 ) higher $P$. nyererei ancestry proportions in $P$. sp. "nyererei-like" and higher P. pundamilia ancestry proportions in P. sp. "pundamilia-like", for instance due to differential sorting of the parental haplotypes, or 2) similar selection pressures on alleles forming part of a shared pool of standing variation (i.e. already present in the Mwanza Gulf Pundamilia population before the admixture event), or 3) ongoing gene flow between the two redbacked species and between the two species with blue male nuptial coloration. More than half of the genomic regions that are highly differentiated between the species in both pairs show excess allele sharing between the two blue and the two red-backed species. This indicates that in these shared HDRs positive selection acts on nyererei-derived alleles in P. sp. "nyererei-like" and on pundamilia-derived alleles in P. sp. "pundamilia-like". It thus seems likely that $P$. nyererei alleles introduced into the ancestral Pundamilia of the north-western Mwanza Gulf in the inferred hybridization event (Meier, et al. 2017b) facilitated evolutionary response to divergent natural and sexual selection pressures between microhabitats. For example, alleles associated with red male nuptial coloration, 
zooplanktivory, and/or occupation of the deep water habitat are the aspects in which P. sp. "nyerereilike" resembles $P$. nyererei, and introduction of alleles related to these traits likely facilitated the evolution of $P$. sp. "nyererei-like".

We identified 41 candidate regions of parallel selection in the two species pairs, whereby in the younger species pair, divergent selection appears to act on the genetic variation derived from the hybridization event. They overlap with 92 genes with potential roles in sensory system, morphological, coloration, trophic and immune system differences between the species. Van Rijssel et al., (2018) showed that some of the parallel morphological differences between the species in the two species pairs are currently under disruptive selection in both species pairs. However, pinpointing the genes underlying these morphological traits requires further research. The candidate gene of parallel selection with most information available is the red-sensitive (LWS) opsin gene. The LWS opsin gene is under divergent selection between these species as the light environment is more red-shifted in deeper water where $P$. nyererei and P. sp. "nyererei-like" occur compared to the shallow-water habitat of $P$. pundamilia and $P$. sp. "pundamilia-like" (Seehausen, et al. 2008). In addition, differences in color vision may modulate the female preferences for differently colored males (Kamijo, et al. 2018) and thus contribute directly to reproductive isolation between the species. We demonstrate that the region around the LWS gene is highly differentiated between the species in both species pairs. The differences in allele frequencies between the species in the two pairs are remarkably parallel not just at the LWS gene but also at nearby genes, whereas they are not parallel in intergenic regions. In the entire region, genes are strongly enriched for SNPs with parallel allele frequency differences in the two species pairs, suggesting a role for parallel divergent selection on admixture-derived allelic variation in the large region containing three opsin genes (LWS, SWS2 $a, S W S 2 b)$ and associated regulatory regions.

\section{Conclusions}

Here, we studied a very young species pair that emerged from admixed ancestry of two older species that had evolved under parallel selection pressures. Despite their very recent origin and ongoing gene flow, the genome-wide average differentiation between the fully sympatric younger species is significant and many genomic regions are highly differentiated. Some of those differentiated regions arose through divergent selection on haplotypes from the different parental species in genomic regions that are also among the most strongly differentiated genomic regions in the older species pair. This finding implicates an important role of hybridization in the generation of genetic variation that facilitates rapid response to divergent natural and sexual selection through differential sorting of admixture-derived haplotypes with multiple co-adapted alleles. In addition, many more genomic regions are divergent between the species in the younger species pair that do not show evidence of 
divergent selection in the older species pair. As supported by assortative mating between the younger and the older red-backed species in the laboratory, this finding shows that the younger species are not merely replicas of the original species, but instead are largely independent species. This independence may facilitate the eventual return to sympatry between the younger and the older species. Hybrid parallel speciation may thus be a process that facilitates the buildup of species richness in young adaptive radiations. 


\section{Materials and Methods}

\section{Samples and sequencing}

Wild males of $P$. nyererei, P. sp. "nyererei-like", P. pundamilia, and P. sp. "pundamilia-like" were collected with gill nets and angling at Makobe Island in the Speke Gulf and at Python Island in the north-western Mwanza Gulf in 2010 (Fig. 1, Meier et al., 2017b). The protocol for obtaining wholegenome sequencing data is detailed in Meier, et al. (2017b). Briefly, PCR-free libraries (Kozarewa, et al. 2009) were produced from phenol-chloroform extracted DNA. To avoid any sequencing lane effects and to get an even read representation, all libraries were pooled after ligation of unique barcodes and sequenced together on four Illumina HiSeq 3000 lanes.

Local alignment against an anchored version of the Pundamilia nyererei reference genome (Brawand, et al. 2014; Feulner, et al. 2018) was performed with Bowtie 2 (Langmead and Salzberg 2012), and variant calling and genotyping was conducted with Haplotype Caller (GATK v. 3. 5, McKenna, et al. 2010) following the best practice recommendations of GATK. To account for potential Illumina barcode switching issues (Sinha et al., 2017), we set the "contamination_fraction_to_filter" parameter in HaplotypeCaller to 0.1 , to ignore $10 \%$ of the reads per allele of each genotype for genotype calling. In addition, we removed heterozygote genotypes with strongly unbalanced distribution of reads per allele by excluding genotypes failing a binomial test at a p-value threshold of 0.001 (custom Python script "allelicBalance.py"). To avoid potential paralogous regions, we excluded sites with a mean depth per individual greater than 1.5 times the interquartile range from the mean (i.e. 28.5, custom bash script "removeTooHighDepthSites.sh"), and sites with excess heterozygosity over all 16 individuals using the vcftools option "--hardy" and a p-value cutoff of 0.01 . We also excluded sites based on the GATK variant annotations, including the fisher strand test, mapping quality and the read position rank sum test (FS>60, MQ<40, MQRankSum<-12.5 and ReadPosRankSum<-8). Genotypes with less than ten reads were set as missing and sites with more than $50 \%$ missing data were excluded. If not otherwise indicated, all filtering steps were performed with vcftools v. 0.1.15 (Danecek, et al. 2011).

\section{Measures of nucleotide diversity and selection}

To study divergence and nucleotide diversity patterns along the genome, we used a sliding window approach on the whole-genome dataset. All statistics were calculated for non-overlapping $20 \mathrm{~kb}$ windows. Absolute divergence $\left(d_{\mathrm{XY}}\right)$, nucleotide diversity $(\pi)$, and the number of segregating sites $(S)$ in each population were calculated with a modified version of a Python script by Martin, et al. (2015). For both species pairs separately, we calculated the difference in nucleotide diversity between blue and red species, which is expected to be large if a selective sweep led to locally reduced diversity in 
one of the species. We also calculated Tajima's D using only sites without missing data in each species with the formula by Tajima (1989).

In order to identify regions of elevated relative divergence, we calculated pairwise locus-specific $\mathrm{F}_{S T}$ values between the species at each of the two islands. For each pairwise comparison, we created a dataset of bi-allelic SNPs sequenced in all eight individuals of the compared populations. We calculated within- and between-group variance components for each SNP with Arlequin v. 3.5 .2 (Excoffier and Lischer 2010) using a locus-by-locus AMOVA including an individual level. We then calculated weighted average $\mathrm{F}_{\text {ST }}$ values for windows of 15 SNPs using the ratio of average variances across all SNPs in the sliding window as recommended by Bhatia, et al. (2013), i.e. as the mean variance between groups divided by the sum of the mean variance between groups, the mean variance within groups, and the mean individual variance. Windows of 15 SNPs provide a good balance between fine-scale genomic resolution and low stochastic variation by averaging over multiple variants (Malinsky, et al. 2015). In addition, using windows of fixed number of variants as opposed to windows of fixed size provides the advantage that the variance is similar in all windows.

As additional measures of divergent selection, we used two extended haplotype length-based selection statistics (EHL), the cross-population extended haplotype homozygosity statistic (XP-EHH, Sabeti, et al. 2007) and the integrated haplotype score (iHS, Voight, et al. 2006). IHS compares the lengths of different haplotypes at the same genomic position within a population. It increases during ongoing sweeps as the sweeping haplotype is much longer than the other haplotypes. XP-EHH compares the lengths of haplotypes between populations and has most power to detect selection when the haplotype under selection is near fixation in one population and polymorphic in the other population. Background selection in regions of low recombination would lead to longer haplotypes in both species and would thus not increase XP-EHH (assuming conserved recombination rates, which is likely true in this case). To compute these EHL statistics, we first phased the dataset using fastPHASE (Scheet and Stephens 2006) assuming 4 clusters (K4). We predicted recombination distances between markers based on the linkage map by Feulner, et al. (2018), based on 1,597 SNPs and 212 F2 individuals from a Pundamilia pundamilia x P. sp. "red head" cross. First, we pruned the linkage map for outliers and markers that were less than $20 \mathrm{~kb}$ apart. Then we fitted a cubic smoothing spline to the physical and recombination distances using the R-function "smooth.spline" setting the smoothing parameter (spar) to 0.7 and predicted the recombination positions in $\mathrm{cM}$ for the genomic positions as the first derivative of the "predict.smooth.spline" function. We computed XP-EHH for both sympatric species pairs and iHS for each species with selscan v. 1.1.0b (Szpiech and Hernandez 2014). Given that we do not know the ancestral and derived states of the SNPs, we used absolute iHS values. Similarly, as we do not 
distinguish in which species selection acted, we used absolute XP-EHH values. We normalized both statistics in allele frequency bins of 0.05 across all chromosomes combined as in Voight et al, 2006. Next, we computed the fraction of normalized absolute scores above 2.0 in non-overlapping $20 \mathrm{~kb}$ windows with norm v.1.1.0a (Szpiech, Hernandez 2014). A region with a high fraction of extreme scores is a better indicator of selective sweeps than single high scores (Voight et al., 2006). We identified "outlier windows" as top 5\% windows with the highest fractions of extreme normalized scores among windows with similar number of SNPs (100 bins) using norm v.1.1.0a (Szpiech and Hernandez 2014).

\section{Measures of excess allele sharing}

The gene flow statistic $f_{d}$ is similar to $D$ statistics (ABBA-BABA tests) but is more robust to low numbers of SNPs and thus more suited for gene flow estimates in small genomic regions (Martin, et al. 2015). Like $D, f_{d}$ is also used to measure excess allele sharing between two populations by comparing allele frequencies among four populations. The four populations are required to have a genome-wide average tree of the following topology: ((P1,P2),P3,O). P3 is tested for excess allele sharing with P2 relative to its sister population $\mathrm{P} 1$, and $\mathrm{O}$ denotes an outgroup. In the absence of hybridization, $\mathrm{P} 1$ and P2 should both share equal numbers of alleles with P3. Excess allele sharing between P2 and P3 is usually interpreted as signature of gene flow but could also arise from parallel selection on shared ancestral polymorphisms. The outgroup is used to define the ancestral (A) and derived (B) state of the two alleles. The $f_{d}$ statistic is then calculated as the difference in number of ABBA (P2 and P3 share the derived allele) and BABA (P1 and P3 share the derived allele) counts relative to the expected counts under a scenario of complete mixing between P2 and P3. For more details see Martin, et al. (2015). High $f_{d}$ estimates indicate excess allele sharing and thus likely gene flow between P2 and P3.

We calculated $f_{d}$ using $H$. vittatus from Lake Kivu as outgroup. Assuming that the species at Python Island are sister taxa, we tested for excess allele sharing between the two red-backed species, $P$. nyererei Makobe and $P$. sp. "nyererei-like" at Python ( $f_{d}$ nyer, $\mathrm{P} 1=P$. sp. "pundamilia-like" Python, $\mathrm{P} 2=P$. sp. "nyererei-like", $\mathrm{P} 3=P$. nyererei), and between the two blue species, $P$. pundamilia and $P$. sp. “pundamilia-like" ( $f_{d}$ pund, $\mathrm{P} 1=P$. sp. "nyererei-like" Python, $\mathrm{P} 2=P$. sp. "pundamilia-like" Python, $\mathrm{P} 3=P$. pundamilia Makobe). To minimize false signatures of introgression due to low numbers of ancestryinformative sites, we calculated $f_{d}$ statistics only for sliding windows where the sum of $A B B A$ and $B A B A$ counts was at least five. As fixed ABBA or BABA patterns are rare, most sites fit the ABBA or BABA patterns only partially and are thus also only counted partially. As an example, if the allele frequencies are $\mathrm{p}_{1}=0.5, \mathrm{p}_{2}=0, \mathrm{p}_{3}=0.5$, and $\mathrm{p}_{0}=0$, it would count as $0.25 \mathrm{BABA}$ pattern $\left(\mathrm{C}_{\mathrm{BABA}}=\mathrm{p}_{1} *\left(1-\mathrm{p}_{2}\right) * \mathrm{p}_{3} *\left(1-\mathrm{p}_{\mathrm{o}}\right)\right)$. Therefore, many sites partially fitting the ABBA or BABA pattern are needed to reach a total sum of five. 


\section{Identification of highly differentiated genomic regions}

In order to identify genomic regions of high interspecific differentiation and to assess clustering of high $\mathrm{F}_{S T}$ windows, we applied a discrete state Hidden Markov Model (HMM) approach for the species pairs at Makobe and at Python Island separately.

We performed 10,000 coalescent simulations under the demographic model inferred to best explain the observed data (Meier, et al. 2017b) using fastsimcoal2 (Excoffier, et al. 2013). We used the empirically inferred average recombination rate of $2.3 \times 10^{-8}$ per bp and generation and the mutation rate of $6.6 \times 10^{-8}$ estimated by Recknagel, et al. (2013). We simulated eight $20 \mathrm{~kb}$ sequences corresponding to 4 diploid individuals per species. We calculated pairwise $F_{S T}$ estimates for both species pairs separately averaged across the first 15 SNPs like for the observed data. The distribution of the 10,000 weighted $F_{S T}$ averages was then used as null distribution to compute z-scores for the observed 15 SNP $F_{S T}$ averages. For each observed $F_{S T}$ average, we computed the $p$-value as the quantile of the null distribution. Next, we transformed p-values into z-scores (i.e. normal scores) using the Rfunction "qnorm". The z-scores were approximately normally distributed indicating a good fit of the null model to the observed data.

An HMM analysis was then performed on the z-scores to assign each 15-SNP window to either the "standard" or "high differentiation". We fixed the means to 0 for the "standard state" and to 1.28 ( $p$ value of 0.1 ) for the "high differentiation state" and the standard deviations matching the empirical values. Following Hofer, et al. (2012) and Marques, et al. (2016), transition and emission probabilities were optimized with the Baum-Welch algorithm (6 runs with 1000 iterations each, Baum, et al. 1970) and the most likely sequence of states given the observed z-scores was inferred with the Viterbi algorithm (Viterbi 1967). As in Marques, et al. (2016), we concatenated the chromosomes to increase the information for parameter estimation. We did not find spurious regions of high differentiation resulting from concatenation of the chromosomes, i.e. no erroneous islands extending over multiple chromosomes.

To assess clustering of highly differentiated windows, we concatenated directly adjacent windows assigned to the "high differentiation" state to "highly differentiated genomic regions" (HDR). Genomic regions where HDRs from Makobe and from Python Island overlapped were termed shared HDRs and considered to be candidates of parallel divergence. We used a block-permutation approach to assess if the number and length of these shared HDRs differed from random expectations. We permuted the HMM states for each species pair separately in blocks of 100 windows and recalculated the number and mean lengths of shared HDRs. A block size of 100 windows exceeds the length of $98 \%$ of HDRs 
allowing us to account for the clustered distribution of highly differentiated windows. The permutation procedure was repeated 100 times to get a random distribution of overlap statistics.

Genome-wide average differentiation between the sympatric species outside of the highly divergent genomic regions was assessed by computing mean $\mathrm{F}_{S T}$ values from SNPs without missing data that were pruned for high linkage disequilibrium $\left(r^{2}>0.5\right)$ with plink v.1.07 (using --indep-pairwise 5010 0.5, Purcell, et al. 2007) and vcftools v. 0.1.15 (Danecek, et al. 2011) using a custom script (IdPruning.sh). Average $F_{S T}$ values between the species were computed with an ANOVA performed in Arlequin v. 3.5.2 (Excoffier and Lischer 2010). We computed confidence intervals from 20,000 bootstraps to test if the differentiation was significant.

In order to test the robustness of the detection of HDRs, we additionally used another approach to identify HDRs. We computed window averages for non-overlapping $20 \mathrm{~kb}$ windows and for $50 \mathrm{~kb}$ windows. We identified the windows with $\mathrm{F}_{\mathrm{ST}}$ estimates above the $90^{\text {th }}$ quantile and concatenated consecutive windows. To avoid splitting of HDRs due to short stochastic dips in $\mathrm{F}_{S T}$, we ignored single windows with $\mathrm{F}_{\mathrm{ST}}$ estimates below the threshold within runs of highly differentiated windows. The HDRs were identified both for the Makobe and the Python species pairs and compared to the HDRs identified with the HMM approach based on 15-SNP windows.

To test for genome-wide correlation of differentiation between the two species pairs, we compared FST $_{\text {ST }}$ estimates of the older species pair at Makobe Island with FST $_{\text {T }}$ estimates of the younger species pair at Python Island in the same genomic regions. In addition, we used restriction-site associated DNA (RAD) sequencing data from Meier et al., (2017b) to compare the extent of correlation between the species pairs to the correlation of differentiation with another Pundamilia population from Luanso Island. Luanso Island is approximately $7.5 \mathrm{~km}$ away from Python Island and $37.5 \mathrm{~km}$ from Makobe Island. It harbors a phenotypically polymorphic but undifferentiated population of Pundamilia cichlids and is thus ideal to assess the expected level of correlation without selection (e.g. due to background selection). In addition, we used RAD data from a second sampling location of the younger species pair, Kissenda Island, which is $9.6 \mathrm{~km}$ north of Python Island. We used 20 individuals of $P$. nyererei and 19 individuals of $P$. pundamilia, all sampled at Makobe Island. We included 16 or 22 P. sp. "nyererei-like" and 26 or 22 individuals of P. sp. "pundamilia-like" from Kissenda Island or from Python Island, respectively. Of the Pundamilia population at Luanso Island, we used 53 individuals. We used only sites sequenced in at least 10 individuals per population or species with at least 10 reads per individual. Otherwise, we applied the same filtering steps as detailed in Meier et al., (2017b). Fst values were estimated for all species/population pairs using the site-by-site ANOVA implemented in Arlequin v. 3.5.2 (Excoffier and Lischer 2010). We computed the ratio of average variances (Bhatia et al., 2013) 
across all SNPs in $20 \mathrm{~kb}$ non-overlapping windows both for the RAD dataset and the whole-genome dataset. Windows with less than 3 SNPs were discarded. Correlation of the F estimates was assessed with the Pearson's correlation using the R function "cor.test". To further assess genomic parallelism with the RAD sequencing dataset, we extracted all $20 \mathrm{~kb}$ windows with an $\mathrm{F}_{\mathrm{ST}}$ estimate above 0.2 for the Makobe species pair. We then tested if these windows also exhibit higher $\mathrm{F}_{\mathrm{ST}}$ estimates than null expectations from 1,000 permuted datasets in the younger species pair sampled at Python and Kissenda Island and in the control comparison of each Python species with the Luanso population.

\section{Parallelism at the genomic level}

We tested for parallelism at the genomic level by assessing the predominant tree topologies in $20 \mathrm{~kb}$ windows using TWISST (Martin and Van Belleghem 2017). First, we computed for each $20 \mathrm{~kb}$ window a maximum likelihood tree of all 16 individuals using raxmIHPC-AVX (v. 8.2.4, Stamatakis 2014) and raxmIWindows.py (https://github.com/simonhmartin/genomics_general). Next, we used TWISST to iteratively subsample all combination of a single individual per species (option "complete") and compute the proportion of subtrees matching each of the possible species topologies. With four species, three unrooted tree topologies are possible: the "geography topology", where species of Python Island cluster together and species of Makobe Island cluster together, the "color topology", where $P$. nyererei and $P$. sp. "nyererei-like" cluster together and $P$. pundamilia and $P$. sp. "pundamilialike" cluster together, and the "random topology", where $P$. pundamilia clusters with $P$. sp. "nyerereilike" and $P$. nyererei clusters with $P$. sp. "pundamilia-like". High weights of the "color topology" in a genomic region may be due to parallel selection in the two species pairs.

We determined candidate regions of parallel selection as $20 \mathrm{~kb}$ windows in shared highly divergent regions (SHDRs) with a weight of at least 66\% for the "color topology". A weight of 66\% means that the topology is twice as well supported than the two other topologies together. For these windows, we identified overlapping genes from the NCBI Pundamilia nyererei RefSeq annotation release 101 (https://www.ncbi.nlm.nih.gov/genome/annotation euk/Pundamilia nyererei/101/\#BuildInfo) lifted to the new anchored version of the P. nyererei reference genome (Brawand, et al. 2014; Feulner, et al. 2018) using the UCSC tool liftOver (Hinrichs, et al. 2006) and custom chain files. We searched OrthoDB (Zdobnov, et al. 2017) for orthologs of these candidate genes in Danio rerio, Mus musculus, Rattus norvegicus and Homo sapiens, extracted associated functional annotation for these orthologs and assessed their functional relevance in light of the natural history of Pundamilia speciation. 


\section{Acknowledgements}

Many thanks to Krushnamegh Kunte and Deepa Agashe for the invitation to contribute to the special issue on Genetics of Adaptation. We thank the Tanzania Fisheries Research Institute (TAFIRI) for hosting us during fieldwork and the Tanzania Commission for Science \& Technology (COSTECH) for research permits. We thank Stefan Zoller and Niklaus Zemp from the Genetic Diversity Center (GDC) at ETH Zürich for bioinformatics support. Genomic analyses were performed using the computing infrastructure of the GDC and the Euler computer cluster at ETH Zurich. We also thank Simon Aeschbacher for discussion about divergent and background selection. This research was supported by the Swiss National Science Foundation grants PDFMP3 134657 to OS and LE and 163338 to OS.

\section{Author contributions}

J.I.M. and O.S. designed the study; J.I.M. performed the analyses with assistance of the other authors; D.A.M. explored the gene functions; J.I.M wrote the manuscript with contributions from O.S., L.E., C.E.W., and D.A.M.

\section{References}

Abbott R, Albach D, Ansell S, Arntzen JW, Baird SJ, Bierne N, Boughman J, Brelsford A, Buerkle CA, Buggs R, Butlin RK, Dieckmann U, Eroukhmanoff F, Grill A, Cahan SH, Hermansen JS, Hewitt G, Hudson AG, Jiggins C, Jones J, Keller B, Marczewski T, Mallet J, Martinez-Rodriguez P, Most M, Mullen S, Nichols R, Nolte AW, Parisod C, Pfennig K, Rice AM, Ritchie MG, Seifert B, Smadja CM, Stelkens R, Szymura JM, Vainola R, Wolf JB, Zinner D 2013. Hybridization and speciation. J Evol Biol 26: 229-246.

Barton N 1983. Multilocus clines. Evolution: 454-471.

Baum LE, Petrie T 1966. Statistical inference for probabilistic functions of finite state markov chains. The annals of mathematical statistics 37: 1554-1563.

Baum LE, Petrie T, Soules G, Weiss N 1970. A maximization technique occurring in the statistical analysis of probabilistic functions of markov chains. Ann Mat Stat 41: 164-171.

Bhatia G, Patterson N, Sankararaman S, Price AL 2013. Estimating and interpreting fst: The impact of rare variants. Genome Res 23: 1514-1521.

Bouton N, Seehausen O, van Alphen JJM 1997. Resource partitioning among rock-dwelling haplochromines (pisces : Cichlidae) from lake victoria. Ecology of Freshwater Fish 6: 225-240.

Boyle EA, Li YI, Pritchard JK 2017. An expanded view of complex traits: From polygenic to omnigenic. Cell 169: 1177-1186.

Brawand D, Wagner CE, Li YI, Malinsky M, Keller I, Fan SH, Simakov O, Ng AY, Lim ZW, Bezault E, TurnerMaier J, Johnson J, Alcazar R, Noh HJ, Russell P, Aken B, Alfoldi J, Amemiya C, Azzouzi N, Baroiller JF, Barloy-Hubler F, Berlin A, Bloomquist R, Carleton KL, Conte MA, D'Cotta H, Eshel O, Gaffney L, Galibert F, Gante HF, Gnerre S, Greuter L, Guyon R, Haddad NS, Haerty W, Harris RM, Hofmann HA, Hourlier T, Hulata G, Jaffe DB, Lara M, Lee AP, MacCallum I, Mwaiko S, Nikaido M, Nishihara H, Ozouf-Costaz C, Penman DJ, Przybylski D, Rakotomanga M, Renn SCP, Ribeiro FJ, Ron M, Salzburger 
W, Sanchez-Pulido L, Santos ME, Searle S, Sharpe T, Swofford R, Tan FJ, Williams L, Young S, Yin SY, Okada N, Kocher TD, Miska EA, Lander ES, Venkatesh B, Fernald RD, Meyer A, Ponting CP, Streelman JT, Lindblad-Toh K, Seehausen O, Di Palma F 2014. The genomic substrate for adaptive radiation in african cichlid fish. Nature 513: 375-381.

Bürger R, Akerman A 2011. The effects of linkage and gene flow on local adaptation: A two-locus continent-island model. Theor Pop Gen 80: 272-288.

Burri R 2017. Interpreting differentiation landscapes in the light of long-term linked selection. Evol Lett 1: 118-131.

Burri R, Nater A, Kawakami T, Mugal CF, Olason PI, Smeds L, Suh A, Dutoit L, Bureš S, Garamszegi LZ 2015. Linked selection and recombination rate variation drive the evolution of the genomic landscape of differentiation across the speciation continuum of ficedula flycatchers. Genome Res 25: 1656-1665.

Butlin RK 2005. Recombination and speciation. Mol Ecol 14: 2621-2635.

Carleton KL, Parry JWL, Bowmaker JK, Hunt DM, O. S 2005. Color vision and speciation in lake victoria cichlids of the genus pundamilia. Mol Ecol 14.

Charlesworth B, Morgan MT, Charlesworth D 1993. The effect of deleterious mutations on neutral molecular variation. Genetics 134: 1289-1303.

Conte GL, Arnegard ME, Peichel CL, Schluter D 2012. The probability of genetic parallelism and convergence in natural populations. Proc Biol Sci B 279: 5039-5047.

Coyne JA, Orr HA. 2004. Speciation: Sinauer Associates Sunderland, MA.

Cruickshank TE, Hahn MW 2014. Reanalysis suggests that genomic islands of speciation are due to reduced diversity, not reduced gene flow. Mol Ecol 23: 3133-3157.

Danecek P, Auton A, Abecasis G, Albers CA, Banks E, DePristo MA, Handsaker RE, Lunter G, Marth GT, Sherry ST, McVean G, Durbin R, Genomes Project Analysis G 2011. The variant call format and vcftools. Bioinformatics 27: 2156-2158.

Excoffier L, Dupanloup I, Huerta-Sánchez E, Sousa VC, Foll M 2013. Robust demographic inference from genomic and snp data. PLoS Genet 9: e1003905.

Excoffier L, Lischer HEL 2010. Arlequin suite ver 3.5: A new series of programs to perform population genetics analyses under linux and windows. Mol Ecol Resour 10: 564-567.

Feder JL, Egan SP, Nosil P 2012a. The genomics of speciation-with-gene-flow. Trends Genet 28: 342350.

Feder JL, Gejji R, Yeaman S, Nosil P 2012b. Establishment of new mutations under divergence and genome hitchhiking. Phil Trans R Soc B 367: 461-474.

Feder JL, Nosil P 2010. The efficacy of divergence hitchhiking in generating genomic islands during ecological speciation. Evolution 64: 1729-1747.

Feder JL, Nosil P, Wacholder AC, Egan SP, Berlocher SH, Flaxman SM 2014. Genome-wide congealing and rapid transitions across the speciation continuum during speciation with gene flow. J Hered 105: 810-820.

Feulner PGD, Schwarzer J, Haesler MP, Meier JI, Seehausen O 2018. A dense linkage map of lake victoria cichlids improved the <em>pundamilia</em> genome assembly and revealed a major qtl for sexdetermination. bioRxiv.

Flaxman S, Feder J, Nosil P 2012. Spatially explicit models of divergence and genome hitchhiking. J Evol Biol 25: 2633-2650.

Flaxman SM, Wacholder AC, Feder JL, Nosil P 2014. Theoretical models of the influence of genomic architecture on the dynamics of speciation. Mol Ecol 23: 4074-4088.

Gilman RT, Behm JE 2011. Hybridization, species collapse, and species reemergence after disturbance to premating mechanisms of reproductive isolation. Evolution 65: 2592-2605.

Grant PR, Grant BR 1992. Hybridization of bird species. Science 256: 193-197.

Haesler MP, Seehausen $O$ 2005. Inheritance of female mating preference in a sympatric sibling species pair of lake victoria cichlids: Implications for speciation. Proc Biol Sci B 272: 237-245. 
Hinrichs AS, Karolchik D, Baertsch R, Barber GP, Bejerano G, Clawson H, Diekhans M, Furey TS, Harte RA, Hsu F, Hillman-Jackson J, Kuhn RM, Pedersen JS, Pohl A, Raney BJ, Rosenbloom KR, Siepel A, Smith KE, Sugnet CW, Sultan-Qurraie A, Thomas DJ, Trumbower H, Weber RJ, Weirauch M, Zweig AS, Haussler D, Kent WJ 2006. The ucsc genome browser database: Update 2006. Nucl Acids Res 34: D590-D598.

Hoekstra HE, Nachman MW 2003. Different genes underlie adaptive melanism in different populations of rock pocket mice. Mol Ecol 12: 1185-1194.

Hofer T, Foll M, Excoffier L 2012. Evolutionary forces shaping genomic islands of population differentiation in humans. Bmc Genomics 13.

Hoffmann AA, Rieseberg LH 2008. Revisiting the impact of inversions in evolution: From population genetic markers to drivers of adaptive shifts and speciation? Annu Rev Ecol Evol Syst 39: 21-42.

Johannesson K, Panova M, Kemppainen P, André C, Rolan-Alvarez E, Butlin RK 2010. Repeated evolution of reproductive isolation in a marine snail: Unveiling mechanisms of speciation. Phil Trans R Soc B 365: 1735-1747.

Johnson TC, Kelts K, Odada E 2000. The holocene history of lake victoria. Ambio 29: 2-11.

Kamijo M, Kawamura M, Fukamachi S 2018. Loss of red opsin genes relaxes sexual isolation between skin-colour variants of medaka. Behavioural Processes 150: 25-28.

Keller I, Wagner CE, Greuter L, Mwaiko S, Selz OM, Sivasundar A, Wittwer S, Seehausen O 2013. Population genomic signatures of divergent adaptation, gene flow and hybrid speciation in the rapid radiation of lake victoria cichlid fishes. Mol Ecol 22: 2848-2863.

Kozarewa I, Ning Z, Quail MA, Sanders MJ, Berriman M, Turner DJ 2009. Amplification-free illumina sequencing-library preparation facilitates improved mapping and assembly of ( $\mathrm{g}+\mathrm{c}$ )-biased genomes. Nature Methods 6: 291-295.

Kronforst MR, Hansen ME, Crawford NG, Gallant JR, Zhang W, Kulathinal RJ, Kapan DD, Mullen SP 2013. Hybridization reveals the evolving genomic architecture of speciation. Cell Rep 5: 666-677.

Lande R 2009. Adaptation to an extraordinary environment by evolution of phenotypic plasticity and genetic assimilation. J Evol Biol 22: 1435-1446.

Langmead B, Salzberg SL 2012. Fast gapped-read alignment with bowtie 2. Nature Methods 9: 357U354.

Maan ME, Hofker KD, van Alphen JJM, Seehausen O 2006. Sensory drive in cichlid speciation. Am Nat 167: 947-954.

Maan ME, Seehausen O, Soderberg L, Johnson L, Ripmeester EAP, Mrosso HDJ, Taylor MI, van Dooren TJM, van Alphen JJM 2004. Intraspecific sexual selection on a speciation trait, male coloration, in the lake victoria cichlid pundamilia nyererei. Proc Biol Sci B 271: 2445-2452.

Maan ME, Van Rooijen AMC, Van Alphen JJM, Seehausen O 2008. Parasite-mediated sexual selection and species divergence in lake victoria cichlid fish. Biol J Linn Soc 94: 53-60.

Magalhaes IS, Mwaiko S, Schneider MV, Seehausen O 2009. Divergent selection and phenotypic plasticity during incipient speciation in lake victoria cichlid fish. J Evol Biol 22: 260-274.

Magalhaes IS, Seehausen 0 2010. Genetics of male nuptial colour divergence between sympatric sister species of a lake victoria cichlid fish. J Evol Biol 23: 914-924.

Malinsky M, Challis RJ, Tyers AM, Schiffels S, Terai Y, Ngatunga BP, Miska EA, Durbin R, Genner MJ, Turner GF 2015. Genomic islands of speciation separate cichlid ecomorphs in an east african crater lake. Science 350: 1493-1498.

Marques DA, Lucek K, Haesler MP, Feller AF, Meier JI, Wagner CE, Excoffier L, Seehausen O 2017. Genomic landscape of early ecological speciation initiated by selection on nuptial colour. Mol Ecol 26: 7-24.

Marques DA, Lucek K, Meier JI, Mwaiko S, Wagner CE, Excoffier L, Seehausen O 2016. Genomics of rapid incipient speciation in sympatric threespine stickleback. PLoS Genet 12: e1005887.

Martin SH, Dasmahapatra KK, Nadeau NJ, Salazar C, Walters JR, Simpson F, Blaxter M, Manica A, Mallet J, Jiggins CD 2013. Genome-wide evidence for speciation with gene flow in heliconius butterflies. Genome Res 23: 1817-1828. 
Martin SH, Davey JW, Jiggins CD 2015. Evaluating the use of abba-baba statistics to locate introgressed loci. Mol Biol Evol 32: 244-257.

Martin SH, Jiggins CD 2017. Interpreting the genomic landscape of introgression. Curr Opin Genet Dev 47: 69-74.

Martin SH, Van Belleghem SM 2017. Exploring evolutionary relationships across the genome using topology weighting. Genetics 206: 429-438.

McKenna A, Hanna M, Banks E, Sivachenko A, Cibulskis K, Kernytsky A, Garimella K, Altshuler D, Gabriel S, Daly M, DePristo MA 2010. The genome analysis toolkit: A mapreduce framework for analyzing next-generation DNA sequencing data. Genome Res 20: 1297-1303.

Meier JI, Marques DA, Mwaiko S, Wagner CE, Excoffier L, Seehausen O 2017a. Ancient hybridization fuels rapid cichlid fish adaptive radiations. Nat Comm 8: 14363.

Meier JI, Sousa VC, Marques DA, Selz OM, Wagner CE, Excoffier L, Seehausen O 2017b. Demographic modelling with whole-genome data reveals parallel origin of similar pundamilia cichlid species after hybridization. Mol Ecol 26: 123-141.

Nachman MW, Payseur BA 2012. Recombination rate variation and speciation: Theoretical predictions and empirical results from rabbits and mice. Phil Trans R Soc B 367: 409-421.

Nadeau NJ, Martin SH, Kozak KM, Salazar C, Dasmahapatra KK, Davey JW, Baxter SW, Blaxter ML, Mallet $\mathrm{J}$, Jiggins CD 2013. Genome-wide patterns of divergence and gene flow across a butterfly radiation. Mol Ecol 22: 814-826.

Noor MAF, Bennett SM 2009. Islands of speciation or mirages in the desert? Examining the role of restricted recombination in maintaining species. Heredity 103: 439-444.

Nosil P, Harmon LJ, Seehausen O 2009. Ecological explanations for (incomplete) speciation. Trends Ecol Evol 24: 145-156.

Pfennig DW, Wund MA, Snell-Rood EC, Cruickshank T, Schlichting CD, Moczek AP 2010. Phenotypic plasticity's impacts on diversification and speciation. Trends Ecol Evol 25: 459-467.

Pritchard JK, Pickrell JK, Coop G 2010. The genetics of human adaptation: Hard sweeps, soft sweeps, and polygenic adaptation. Current Biol 20: R208-R215.

Purcell S, Neale B, Todd-Brown K, Thomas L, Ferreira MAR, Bender D, Maller J, Sklar P, de Bakker PIW, Daly MJ, Sham PC 2007. Plink: A tool set for whole-genome association and population-based linkage analyses. Am J Hum Genet 81: 559-575.

Ravinet M, Westram A, Johannesson K, Butlin R, Andre C, Panova M 2016. Shared and nonshared genomic divergence in parallel ecotypes of littorina saxatilis at a local scale. Mol Ecol 25: 287-305.

Recknagel H, Elmer KR, Meyer A 2013. A hybrid genetic linkage map of two ecologically and morphologically divergent midas cichlid fishes Amphilophus spp.) obtained by massively parallel DNA sequencing (ddradseq). G3 Genes Genom Genet 3: 65-74.

Renaut S, Grassa C, Yeaman S, Moyers B, Lai Z, Kane N, Bowers J, Burke J, Rieseberg L 2013. Genomic islands of divergence are not affected by geography of speciation in sunflowers. Nat Comm 4: 1827.

Riesch R, Muschick M, Lindtke D, Villoutreix R, Comeault AA, Farkas TE, Lucek K, Hellen E, SoriaCarrasco V, Dennis SR, de Carvalho CF, Safran RJ, Sandoval CP, Feder J, Gries R, Crespi BJ, Gries G, Gompert Z, Nosil P 2017. Transitions between phases of genomic differentiation during stick-insect speciation. Nat Ecol Evol 1: 0082.

Rieseberg LH 2001. Chromosomal rearrangements and speciation. Trends Ecol Evol 16: 351-358.

Roda F, Liu H, Wilkinson MJ, Walter GM, James ME, Bernal DM, Melo MC, Lowe A, Rieseberg LH, Prentis $P$ 2013. Convergence and divergence during the adaptation to similar environments by an australian groundsel. Evolution 67: 2515-2529.

Roesti M, Moser D, Berner D 2013. Recombination in the threespine stickleback genome-patterns and consequences. Mol Ecol 22: 3014-3027.

Rundle HD, Nagel L, Boughman JW, Schluter D 2000. Natural selection and parallel speciation in sympatric sticklebacks. Science 287: 306-308. 
Sabeti PC, Varilly P, Fry B, Lohmueller J, Hostetter E, Cotsapas C, Xie XH, Byrne EH, McCarroll SA, Gaudet R, Schaffner SF, Lander ES, Consortium IH 2007. Genome-wide detection and characterization of positive selection in human populations. Nature 449: 913-U912.

Samuk K, Owens GL, Delmore KE, Miller SE, Rennison DJ, Schluter D 2017. Gene flow and selection interact to promote adaptive divergence in regions of low recombination. Mol Ecol 26: 4378-4390.

Scheet $P$, Stephens M 2006. A fast and flexible statistical model for large-scale population genotype data: Applications to inferring missing genotypes and haplotypic phase. Am J Hum Genet 78: 629644.

Schluter D, Nagel LM 1995. Parallel speciation by natural selection. Am Nat 146: 292-301.

Seehausen 0 1997. Distribution of and reproductive isolation among color morphs of a rock-dwelling lake victoria cichlid (haplochromis nyererei) (vol 5, pg 195, 1996). Ecology of Freshwater Fish 6: 5966.

Seehausen 0 2004. Hybridization and adaptive radiation. Trends Ecol Evol 19: 198-207.

Seehausen O. 1996. Lake Victoria rock cichlids. Taxonomy, ecology and distribution. Verduijn Cichlids, Zevenhuizen, the Netherlands.

Seehausen 0 2002. Patterns in fish radiation are compatible with pleistocene desiccation of lake victoria and 14600 year history for its cichlid species flock. Proc Biol Sci B 269: 491-497.

Seehausen 0. 2009. Progressive levels of trait divergence along a "speciation transect" in the lake victoria cichlid fish pundamilia. In: Butlin RK, Bridle J, Schluter D, editors. Speciation and patterns of diversity. Cambridge: Cambridge University Press. p. 155-176.

Seehausen O, Lippitsch E, Bouton N, Zwennes H 1998. Mbipi, the rock-dwelling cichlids of lake victoria: Description of three new genera and fifteen new species. Ichthyol Explor Freshw 9: 129-228.

Seehausen O, Terai Y, Magalhaes IS, Carleton KL, Mrosso HDJ, Miyagi R, van der Sluijs I, Schneider MV, Maan ME, Tachida H, Imai H, Okada N 2008. Speciation through sensory drive in cichlid fish. Nature 455: 620-626.

Seehausen O, van Alphen JJM 1998. The effect of male coloration on female mate choice in closely related lake victoria cichlids (haplochromis nyererei complex). Behav Ecol Sociobiol 42: 1-8.

Selz OM, Pierotti MER, Maan ME, Schmid C, Seehausen O 2014. Female preference for male color is necessary and sufficient for assortative mating in 2 cichlid sister species. Behav Ecol 25: 612-626.

Selz OM, Thommen R, Pierotti MER, Anaya-Rojas JM, Seehausen O 2016. Differences in male coloration are predicted by divergent sexual selection between populations of a cichlid fish. Proc Biol Sci B 283.

Soria-Carrasco V, Gompert Z, Comeault AA, Farkas TE, Parchman TL, Johnston JS, Buerkle CA, Feder JL, Bast J, Schwander T, Egan SP, Crespi BJ, Nosil P 2014. Stick insect genomes reveal natural selection's role in parallel speciation. Science 344: 738-742.

Stager JC, Johnson TC 2008. The late pleistocene desiccation of lake victoria and the origin of its endemic biota. Hydrobiologia 596: 5-16.

Stamatakis A 2014. Raxml version 8: A tool for phylogenetic analysis and post-analysis of large phylogenies. Bioinformatics 30: 1312-1313.

Stelkens RB, Pierotti MER, Joyce DA, Smith AM, van der Sluijs I, Seehausen O 2008. Disruptive sexual selection on male nuptial coloration in an experimental hybrid population of cichlid fish. Proc Biol Sci B 363: 2861-2870.

Svensson O, Woodhouse K, van Oosterhout C, Smith A, Turner GF, Seehausen O 2017. The genetics of mate preferences in hybrids between two young and sympatric lake victoria cichlid species. Proc $\mathrm{R}$ Soc B 284.

Szpiech ZA, Hernandez RD 2014. Selscan: An efficient multithreaded program to perform ehh-based scans for positive selection. Mol Biol Evol 31: 2824-2827.

Tajima F 1989. Statistical method for testing the neutral mutation hypothesis by DNA polymorphism. Genetics 123: 585-595.

Turner TL, Hahn MW, Nuzhdin SV 2005. Genomic islands of speciation in anopheles gambiae. PLoS Biol 3: $1572-1578$. 
van Rijssel JC, Moser FN, Frei D, Seehausen O 2018. Prevalence of disruptive selection predicts extent of species differentiation in lake victoria cichlids. Proc Biol Sci 285.

Via S 2012. Divergence hitchhiking and the spread of genomic isolation during ecological speciationwith-gene-flow. Phil Trans R Soc B 367: 451-460.

Via S, West J 2008. The genetic mosaic suggests a new role for hitchhiking in ecological speciation. Mol Ecol 17: 4334-4345.

Viterbi A 1967. Error bounds for convolutional codes and an asymptotically optimum decoding algorithm. IEEE Trans Informat Theory 13: 260-269.

Voight BF, Kudaravalli S, Wen XQ, Pritchard JK 2006. A map of recent positive selection in the human genome. PLoS Biol 4: 446-458.

$\mathrm{Wu} \mathrm{Cl} 2001$. The genic view of the process of speciation. J Evol Biol 14: 851-865.

Yeaman S, Whitlock MC 2011. The genetic architecture of adaptation under migration-selection balance. Evolution 65: 1897-1911.

Zdobnov EM, Tegenfeldt F, Kuznetsov D, Waterhouse RM, Simão FA, loannidis P, Seppey M, Loetscher A, Kriventseva EV 2017. Orthodb v9.1: Cataloging evolutionary and functional annotations for animal, fungal, plant, archaeal, bacterial and viral orthologs. Nucl Acids Res 45: D744-D749.

\section{Figures and Tables}

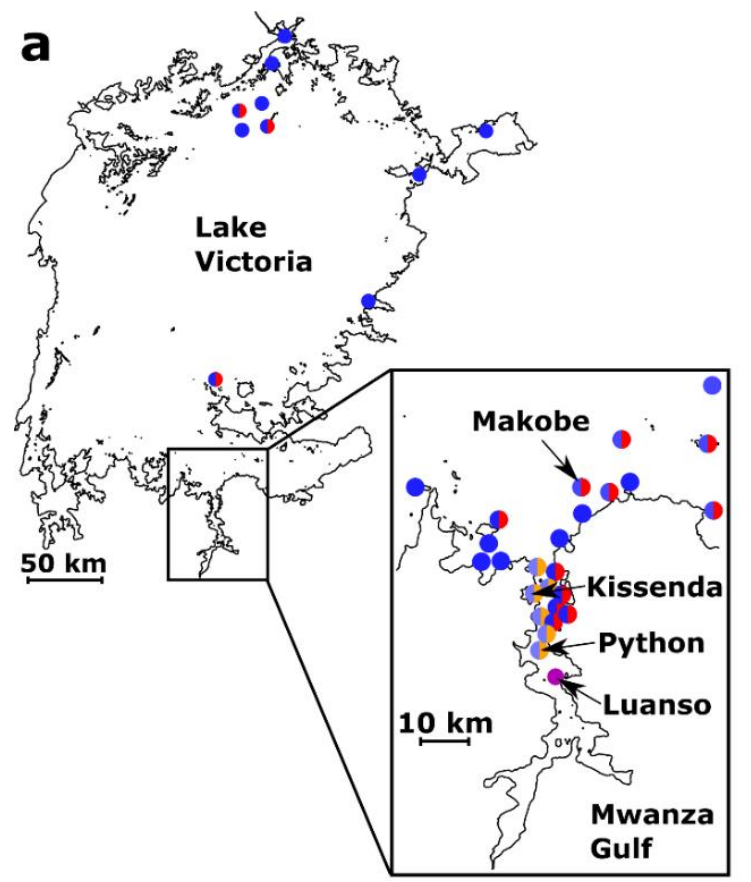

b

older species pair

(e.g. Makobe Island)

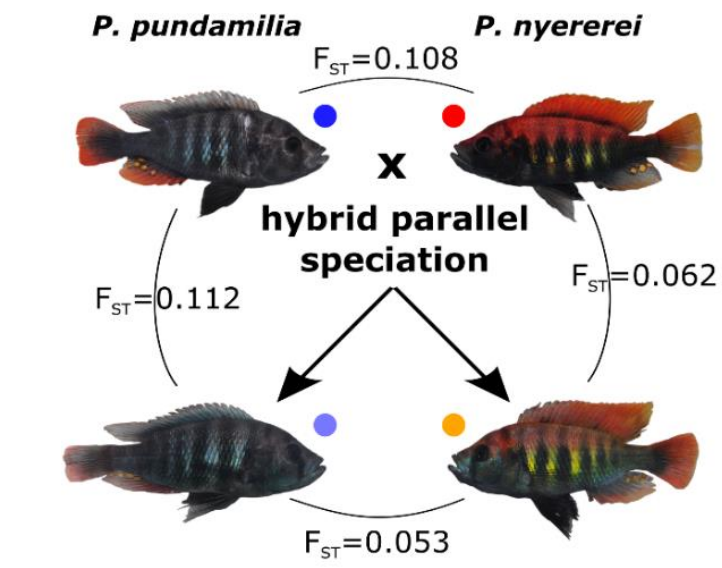

$\begin{array}{lll}P . & \text { sp. "pundamilia-like" } \quad P \text {. sp. "nyererei-like" }\end{array}$

younger species pair

(e.g. Python and Kissenda Island)

Figure 1: Distribution and sampling sites of red and blue Pundamilia cichlids. a) Map of Lake Victoria (modified from Meier et al. 2017) with known records of the older species pair, $P$. pundamilia (blue) and P. nyererei (red), and of the younger species pair, P. sp. "pundamilia-like" (light blue) and $P$. sp. "nyererei-like" (orange). Sites harboring two species are shown as two-colored dots. The inset shows the Mwanza Gulf with the study sites, including Luanso Island (purple), which harbors a colorpolymorphic Pundamilia population that is not differentiated. b) Photos of representative males of each species at the two main study sites, Makobe and Python Island, and mean Fst estimates between the species. 


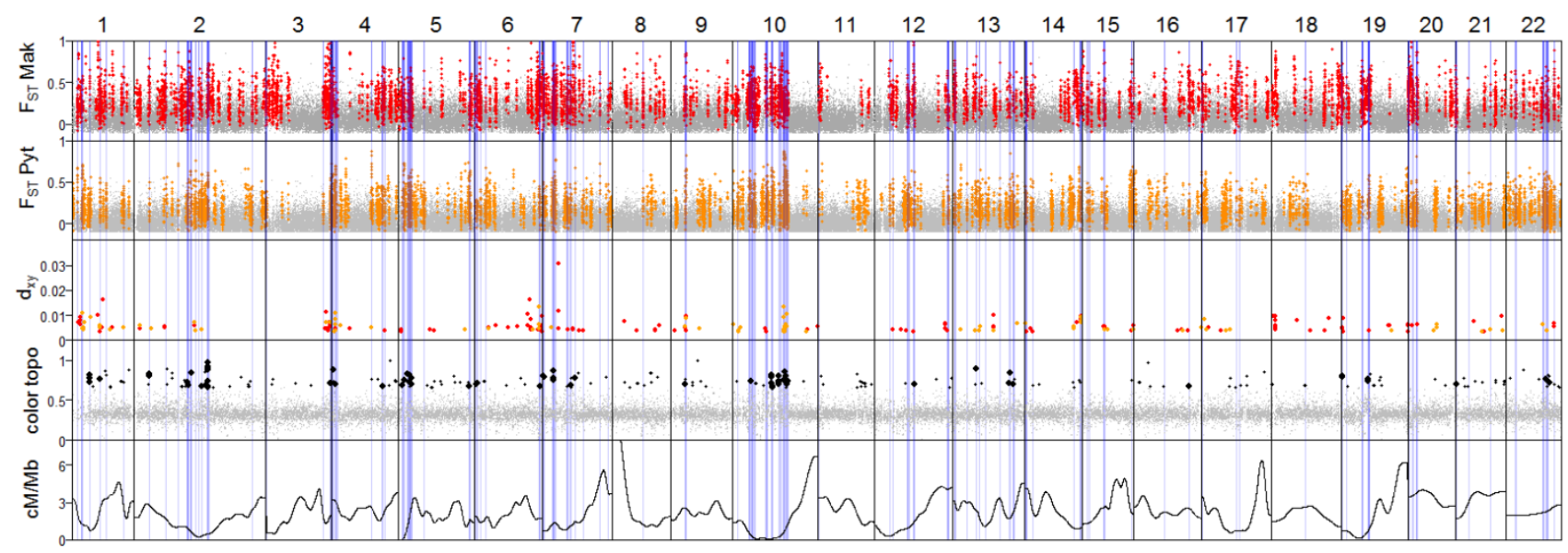

Figure 2: Genomic regions that are highly differentiated in the younger and the older species pair are numerous and widespread across the genome. $\mathrm{F}_{\mathrm{ST}}$ averages of 15-SNP windows between the older species pair (Makobe, top row) and the younger species pair (Python, second row) are shown along the chromosomes (linkage group numbers from Feulner, et al. (2018)), whereby windows assigned to the "high differentiation state" are shown in red (Makobe) or orange (Python). Light blue vertical bars indicate the locations of shared HDRs. Other rows show $d_{x y}$ outliers for Makobe (red) and Python (orange), weights of the "color topology", indicating how strongly the species cluster by male nuptial coloration, and predicted recombination rates in centimorgans per megabase $(\mathrm{cM} / \mathrm{Mb})$. Color topology windows (color topology weight $>66 \%$ ) indicating strong genomic parallelism between the species pairs are shown in black. The 71 color topology windows highlighted with larger black symbols coincide with shared HDRs and are thus considered candidates of parallel divergent selection on shared genetic variation. 


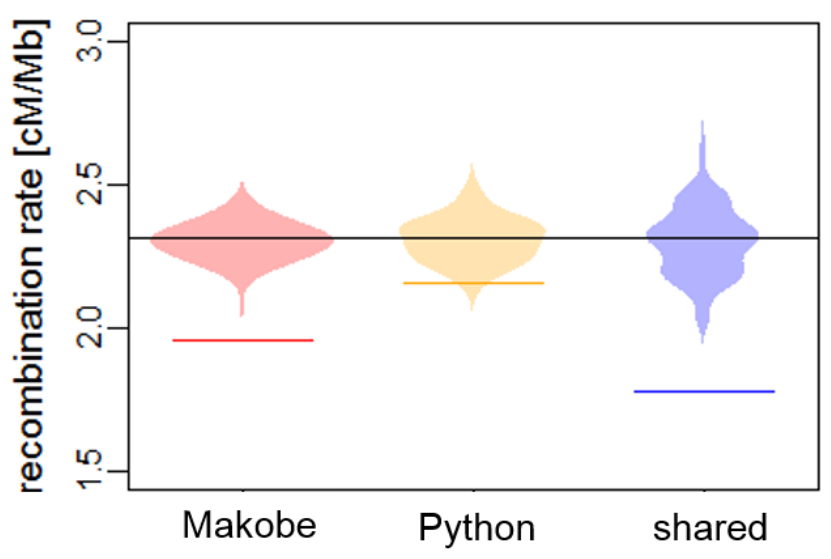

Figure 3: Highly differentiated genomic regions are associated with low recombination rates. The mean recombination rate in highly differentiated regions (HDRs) unique to the Makobe species pair (red horizontal line), unique to the Python species pair (orange line), or shared by both (blue line) are significantly lower than block-permuted null distributions (bean plots, $p$-values: Makobe HDR: $<0.01$, Python HDR: 0.02 , shared HDR: $<0.01$ ). The black horizontal line indicates the overall mean recombination rate. 
a

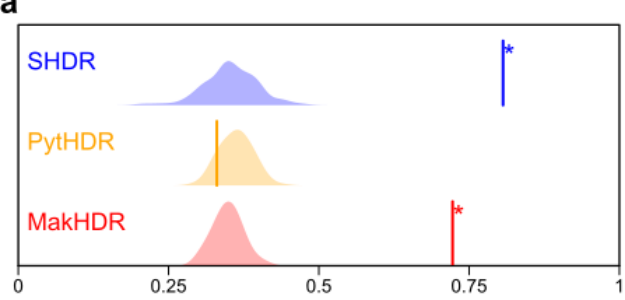

\%HDRs with additional evidence for selection at Makobe b

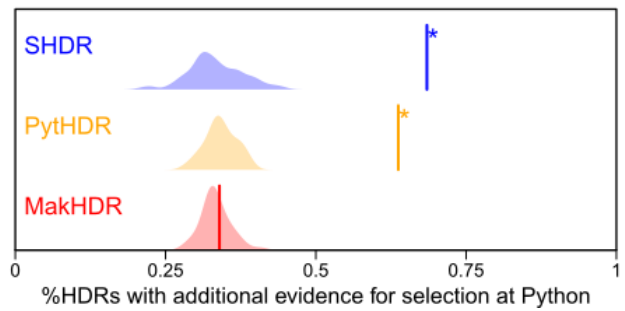

c

\begin{tabular}{|c|c|c|}
\hline & Makobe selection statistics & Python selection statistics \\
\hline & $\mathrm{d}_{\mathrm{xy}} / \mathrm{EHL} / \Delta \pi / \mathrm{T}_{\mathrm{D}}$ & $\mathrm{d}_{\mathrm{xy}} / \mathrm{EHL} / \Delta \pi / \mathrm{T}_{\mathrm{D}}$ \\
\hline SHDR & $\cdot 1 \cdot 1 \cdot 1 \cdot$ & $\cdot 1 \cdot 1 \cdot 1 \cdot$ \\
\hline PytHDR & $-1-1 \cdot 1 \cdot$ & $\cdot 1 \cdot 1 \cdot 1 \cdot$ \\
\hline MakHDR & $\cdot 1 \cdot 1 \cdot 1 \cdot$ & $-1-1-1-$ \\
\hline
\end{tabular}

d

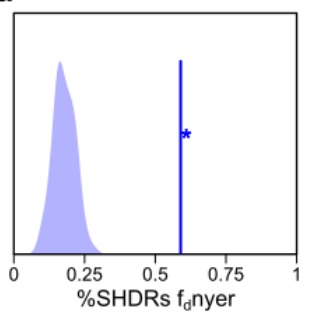

e

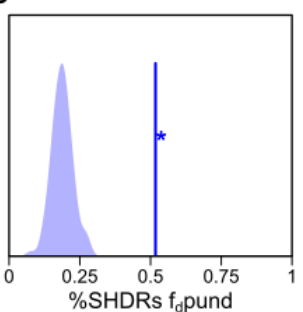

f

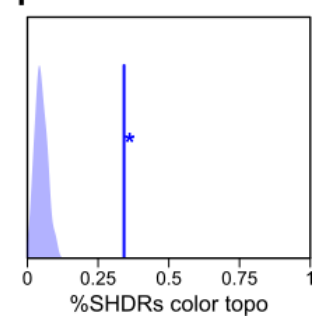

Figure 4: Enrichment of HDRs with evidence for selection. In each panel except for (c), we show the percentages of HDRs with significant statistics (vertical lines) compared to block-permuted null distributions (density plots). In (a) and (b), we show the proportion of HDRs with additional evidence for selection in the Makobe (a) or Python (b) species pair among HDRs unique to the older species pair at Makobe Island (MakHDR, red), among HDRs unique to the younger species pair at Python Island (PytHDR, orange), and among shared HDRs (SHDR, blue). a) At Makobe Island, the proportion of MakHDRs and SHDRs with additional evidence for selection differs strongly from the null distributions, confirming the action of selection generating elevated differentiation in these regions. In contrast, the proportion of PytHDRs with signatures of selection in the Makobe species pair is not different from null expectation, confirming the absence of selection in the Makobe species pair at genomic regions that are highly differentiated only in the Python Island species pair. b) At Python Island, we see a parallel pattern to that in (a): PytHDRs and SHDRs are enriched for selection statistics in the Python species pair, but no such enrichment is found in the MakHDRs, providing evidence that selection in HDRs unique to one species pair is restricted to that species pair. c) Most selection statistics of a given species pair only show enrichment in the HDRs of that species pair. We here show significant enrichment (dot) if the proportion of HDRs spanning an outlier window is higher than the null distribution; the minus symbol indicates non-significant enrichment (see Fig. S6 for more details). Selection statistics include $d_{x y}$, extended haplotype length tests (EHL, including iHS for both species and XP-EHH), difference in nucleotide diversity between the species $(\Delta \pi)$, and low Tajima's $D\left(T_{D}\right)$ in one or both species. $\mathbf{d}$-f) Shared HDRs show evidence for parallel selection leading to sorting of admixture-derived allelic variation in the younger species pair. This is shown by the highly significant proportion of SHDRs that show excess allele sharing between $P$. nyererei and $P$. sp. "nyererei-like" ( $\mathrm{d}$, $\mathrm{f}_{\mathrm{d} n y e r}$ ) or between $P$. pundamilia and $P$. sp. "pundamilia-like" (e, fapund). Similarly, the proportion of SHDRs containing at least one "color topology window", whereby species of the same male nuptial coloration cluster together, strongly exceeds null expectations ( $f$, see also Fig. S12). 


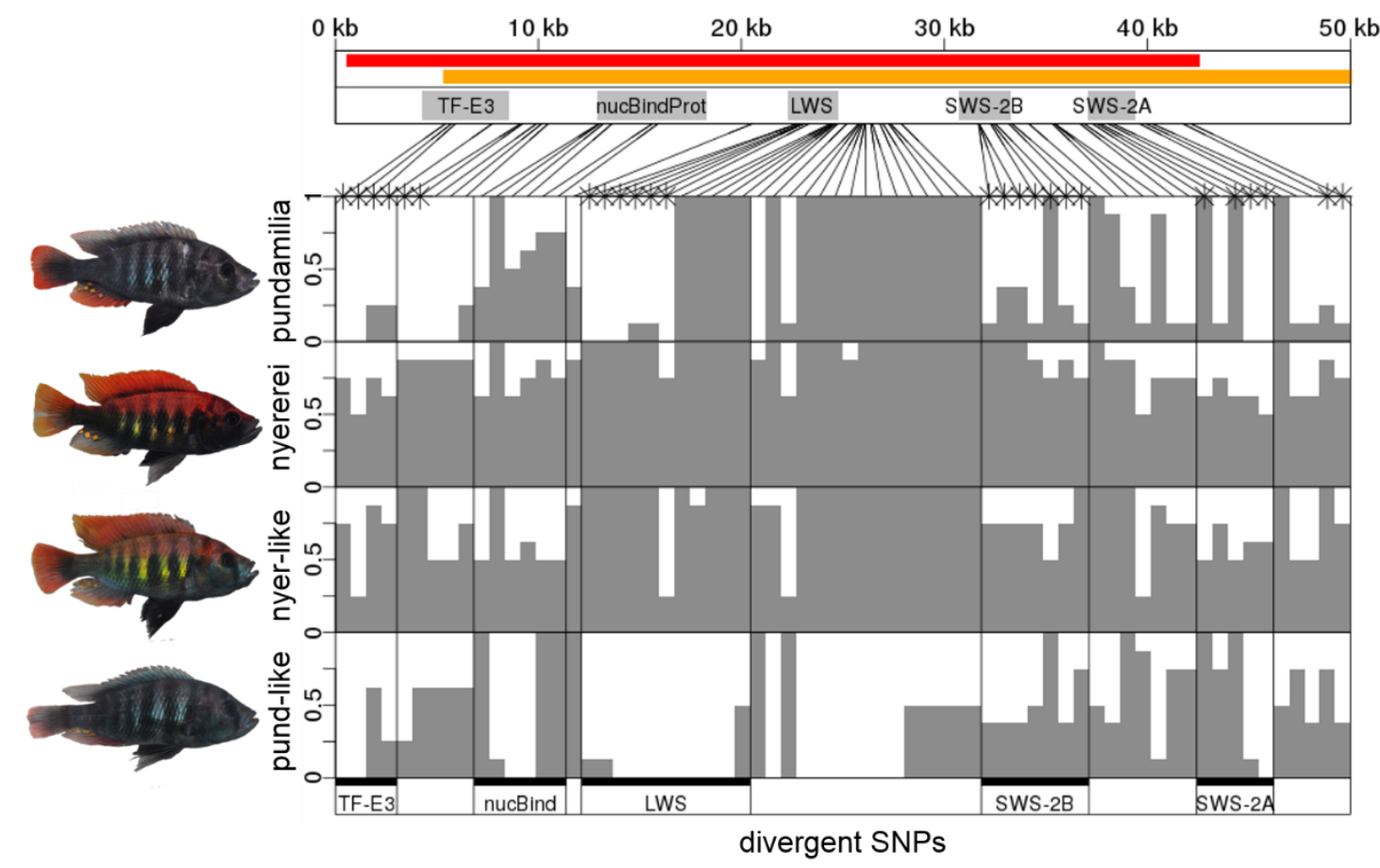

Figure 5: Allele frequency differences in the genomic region of the long wavelength-sensitive (LWS) opsin gene. The region around the LWS opsin gene is located on linkage group 13 (Feulner, et al. 2018) starting at position 26,306,571, corresponding to the beginning of scaffold 177 of the reference assembly of Brawand et al. (2014). The red and orange horizontal bars show the positions of the highly differentiated regions at Makobe and Python Island, respectively, whereas the light grey rectangles indicate gene positions. The first gene codes for the E3-like transcription factor (TF-E3), the second gene for a guanine nucleotide binding protein (nucBindProt), and the three remaining genes are cone opsin genes for red vision (LWS), blue vision (SWS-2A) and violet vision (SWS-2B). The lower panel shows allele frequencies in $P$. pundamilia (pundamilia) and $P$. nyererei (nyererei) at Makobe Island, and $P$. sp. "nyererei-like" (nyer-like) and $P$. sp. "pundamilia-like" (pund-like) at Python Island. Each vertical bar represents an individual SNP with an allele frequency difference between the species at Makobe and or Python Island of at least 0.4. The frequency of the major allele in $P$. nyererei is shown (in grey) for each species ranging from 0 (allele putatively absent) to 1 (allele putatively fixed). Sites with parallel allele frequency differences between the species at Makobe and Python Island are highlighted with stars on top. Note that 21 of 25 parallel sites are located in genes, whereas intergenic regions contain mostly sites with allele frequency shifts that are not parallel between the older and the younger species pair. 
Table 1: Properties of 15-SNP windows assigned to the high differentiation state (HD) and of genomic regions of high differentiation (HDR) between $\boldsymbol{P}$. nyererei and $\boldsymbol{P}$. pundamilia at Makobe (older species pair) and $\boldsymbol{P}$. sp. "nyererei-like" and $\boldsymbol{P}$. sp. "pundamilia-like" at Python Island (younger species pair), as identified with the HMM.

\begin{tabular}{llllllll}
\hline & $\begin{array}{l}\text { Number of HD } \\
\text { windows / total }\end{array}$ & $\begin{array}{l}\text { Mean } \mathrm{F}_{\mathrm{ST}} \text { in } \\
\text { HD / other } \\
\text { windows }\end{array}$ & $\begin{array}{l}\text { Count } \\
\text { of } \\
\text { HDRs }\end{array}$ & $\begin{array}{l}\text { Mean } \\
\text { length } \\
\text { of HDRs }\end{array}$ & $\begin{array}{l}\text { Mean } \\
\text { window } \\
\text { counts } \\
\text { per HDR }\end{array}$ & $\begin{array}{l}\text { Cumula- } \\
\text { tive } \\
\text { length of } \\
\text { HDRs }\end{array}$ & $\begin{array}{l}\text { HDR overlapping } \\
\text { with at least one } \\
\text { HDR in the other } \\
\text { species pair }\end{array}$ \\
\hline Makobe & $8,709 / 111,901(7.8 \%)$ & $0.296 / 0.066$ & 346 & $268 \mathrm{~kb}$ & 25 & $93.0 \mathrm{Mb}$ & $103(29.8 \%)$ \\
Python & $9,811 / 136,961(7.2 \%)$ & $0.207 / 0.031$ & 365 & $196 \mathrm{~kb}$ & 27 & $71.8 \mathrm{Mb}$ & $105(28.8 \%)$ \\
\hline
\end{tabular}

